\title{
The importance of microstructure in electrochemical jet processing
}

\author{
Alistair Speidel ${ }^{1}$, Jonathon Mitchell-Smith ${ }^{1}$, Ivan Bisterov ${ }^{1}$, Adam T. Clare $^{1^{*}}$
}

${ }^{1}$ Advanced Component Engineering Laboratory (ACEL), Advanced Manufacturing Technology Group, Faculty of Engineering, University of Nottingham, Jubilee Campus, NG8 1BB, United Kingdom.

Corresponding author email: adam.clare@nottingham.ac.uk

Keywords: electrochemical jet processing, microstructure, topography, surface modification, process response

\section{Abstract}

Electrochemical jet processing (EJP) is an athermal technique facilitating precision micromachining and surface preparation, without recast layer generation. The role of the microstructure in determining machining characteristics has been largely overlooked. In this study, we show that in order to optimise EJP for a given material, fundamental material factors must be considered to ensure the desired near-surface response in terms of metallurgy, topography and dimensional accuracy. In this work, specimens have been prepared from the same feedstock material (brass, $\mathrm{Cu} 39 \mathrm{Zn} 2 \mathrm{~Pb}$ ), to appraise the role of microstructure in the determination of key removal characteristics, such as resultant topography, removal efficiency and form. Topography is shown to be highly dependent upon microstructure across large current density ranges, whereby the phase ratio is generally the dominant amplitude-defining material property, where preconditions with divergent ratios result in lower amplitudes. The microstructure, specifically the phase ratio, significantly changes the form, where predominantly single-phase conditions result in deeper and narrower features (up to $15 \%$ deeper compared with as-received condition). In addition, removal efficiency is greater (by 6\%) at low current density for small grained dual-phase conditions, than for predominantly single-phase, due to erosion complementing anodic dissolution. Mechanisms are discussed for these removal phenomena and used to inform industrial practice. 


\subsection{Introduction}

Electrochemical jet processing (in machining mode) (EJP/EJM) is a non-conventional process that exploits anodic dissolution and was investigated by Ippolito et al. (1981) for the purpose of material transfer, where the process physics have been described in detail by Kozak et al. (1996). Furthermore, EJP was demonstrated by Natsu et al. (2007) to be an ideal technique for precision micromachining and surface texturing of metals. Material removal in electrochemical machining (ECM) is achieved by anodic dissolution, as opposed to thermal or shear-based methods, and thus no white layer or residual stress is induced in the surface, making EJP ideal for high-value applications, where surface integrity is a primary concern.

Localised anodic dissolution is accomplished by potential difference between a workpiece (anode) and nozzle (cathode), which expels a jet of electrolyte. The electrolyte is directed at a supercritical velocity, such that a radial thin-film forms around the jet impingement area, where the formation is described in detail by White (2010). Electrical current is limited in this region due to the relatively high resistance of the film, thus the current density, $J$, is largely confined beneath the nozzle, giving rise to localised machining. As a result, detailed patterning and texturing can be generated through planar and three-dimensional nozzle motion, without the need of a mask or feature-specific tooling, which has been undertaken by Hackert-Oschätzchen et al. (2012), for the generation of microchannel geometries. In EJP, an applied mean current density, $J_{n o z}$, is generally used in describing the total current, $I$, across the area, $A$, of the conductive electrolyte jet. More accurately, localised current density across 
the electrolyte jet, follows a Gaussian-type distribution, described by Yoneda and Kunieda (1995), which defines the feature form.

Planar feature processing involves nozzle translation, however this is known to affect the resulting topography as the machining zone is exposed to the lowest part of the current density distribution, although Kawanaka and Kunieda (2015) showed that this can be controlled through application of a pulsed power supply and manipulation of process parameters. In addition, Mitchell-Smith et al. (2018a) also reduced deleterious translation effects through manipulation of the angle of nozzle address. Fundamentally, the current density distribution cannot sufficiently define the microscale surface resulting from EJP, as the rate of dissolution at a given processing point is dependent not only on the localised current density, but also materialdependent factors detailed by Kozak and Zybura-Skrabalak (2016). These include passivation anisotropy, ease of constituent element oxidation, as well as the difference in phase machinability that is dependent on electrochemical equivalent (ECE). These serve to define the microscale topography and are therefore important in understanding material-process interaction.

In light of this, the role of material precondition in topography and feature generation in EJP, in order to inform process considerations, has largely been overlooked since Speidel et al. (2016) showed that heat treatments and resulting microstructural differences change the processing response of the material to EJP. In difficult-tomachine metal matrix composites, increasing precipitate grain size has been shown to increase surface amplitude in EJP, due to the inert response of the ceramic 
reinforcements to the process. Hackert-Oschätzchen et al. (2013) showed that this influences the resulting topography. In addition, Kunieda et al. (2016) reported on the reduction in material removal rates caused by increasing grain size, through decreasing erosive precipitate wash-out. However, this has not been fully appraised in the EJP of dual-phase alloys, where both phases can be anodically dissolved within common EJP current density parameters $\left(<300 \mathrm{~A} / \mathrm{cm}^{2}\right)$, using $\mathrm{pH}$ neutral electrolytes. In particular, Pickering and Wagner (1967) reported that where there is an element of the alloy that is significantly more oxidisable, this may be stripped preferentially from a given phase. Specifically regarding brass, the material studied here, Pickering and Byrne (1969) observed Cu evolution only at high potentials. For this to occur in EJP, the composition of the near-surface would be altered and it is therefore worthy of further investigation.

Prior work in conventional ECM of steels undertaken by Krishnaiah-Chetty and Radhakrishnan (1981) established that the post-machined surface is defined by the morphology, shape and size of the underlying grain structure, and not only the ECE of the component phases. In addition, Haisch et al. (2004) also reported differences in resulting surface chemistry after investigating surface film constituents during highrate anodic dissolution in steel, found to be amorphous iron oxides with embedded carbides from the metallic matrix. More recently, Burger et al. (2012) studied selective element depletion in the precise electrochemical machining (PECM) of a single crystal Ni-alloy. It was indicated that low current density machining leads to inhomogeneous elemental dissolution, which resulted in topographic undulations in the investigation. This effect was also investigated in a different additively manufactured Ni-alloy by Wang et al. (2017), where undulations were observed to correlate with dendritic 
structures and layer bands, although topographic sensitivity to microstructure was reduced at increased current density. As part of an effort to define a process signature, Klocke et al. (2018) identified different causes of material modifications at the nearsurface resulting from ECM, relating both to electrolyte flow and material microstructural properties, such as passivation, selective dissolution, and pitting, summarising within the investigation that achievable surface topographies strongly depend on the input microstructure.

Considering the results of prior investigations in conventional ECM processes, it is probable that the microstructure will play an important role in determining the nearsurface characteristics in EJP, at least over some of the process window. However, these effects are likely to be different when compared with conventional ECM processing, as impinging jet process can attain current densities significantly greater (>100 A/cm²), with rapid flow velocities. In addition, a fundamental study on material response in EJP, for a dual-phase alloy in different supply conditions, has yet to be undertaken.

In this study, we demonstrate that microstructural factors affect the resultant nearsurface chemistry, topography and form and that through careful consideration the processing conditions can be informed in order to realise surface design. 


\subsection{Methodology}

\subsection{Surface processing}

To appraise the effect of current density on the resulting EJP surface for each given material precondition, two modes of machining were undertaken: the point approach and the vector approach. An array of pits and striations were machined for each material condition, using a three-axis CNC EJP platform detailed in previous work by Mitchell-Smith et al. (2018b). Machining parameters are listed in Table 1. As the resulting surface after EJP is known to be affected by the low current density area of the distribution as the electrolyte jet traverses the workpiece, the point approach was considered important to understanding the mechanisms of surface generation as well as material removal. In point machining, the central region is exposed only to the greatest part of the current density distribution therefore generating an accurate topographic response to the applied current density. Three pits were drilled into each material precondition at each current density. The total charge passed, $Q$, was controlled in the process (uncertainty $\pm 1.95 \mu \mathrm{C}$ ) as the product of applied current, $I$, and time, $t$, and is listed adjacent to the approximate supplied current density. The vector approach, commonly employed by EJP practitioners, was also appraised through the machining of striations, in order to characterise surface generation in a more applicable machining environment. Nozzle traverse velocity, $v_{h}$, was kept constant throughout striation machining $(500 \mu \mathrm{m} / \mathrm{s})$. 
Table 1: EJP parameters used in this study for pit and planar processing.

\begin{tabular}{|c|c|c|c|}
\hline Universal Parameters & \multicolumn{3}{|l|}{ Value } \\
\hline Nozzle internal diameter $(\mu \mathrm{m})$ & \multicolumn{3}{|l|}{510} \\
\hline Inter-electrode gap $(\mu \mathrm{m})$ & \multicolumn{3}{|l|}{500} \\
\hline Electrolyte Concentration $\left(\mathrm{NaNO}_{3}, \mathrm{M}\right)$ & \multicolumn{3}{|l|}{2.3} \\
\hline Electrolyte jet velocity $(\mathrm{m} / \mathrm{s})$ & \multicolumn{3}{|l|}{25.0} \\
\hline \multirow[t]{11}{*}{ Pit machining parameters } & $\begin{array}{l}\text { Approximate mean current } \\
\text { density, } J_{n o z}\left(A / \mathrm{cm}^{2}\right)\end{array}$ & $\begin{array}{l}\text { Charge total per pit } \\
\text { (C) }\end{array}$ & $\begin{array}{l}\text { Dwell time } \\
\text { (ms) }\end{array}$ \\
\hline & 20 & 0.546 & 14000 \\
\hline & 50 & 0.566 & 5600 \\
\hline & 100 & 0.534 & 2667 \\
\hline & 150 & 0.558 & 1806 \\
\hline & 200 & 0.560 & 1366 \\
\hline & 250 & 0.560 & 1098 \\
\hline & 300 & 0.563 & 918 \\
\hline & 350 & 0.554 & 778 \\
\hline & 400 & 0.559 & 682 \\
\hline & 450 & 0.532 & 589 \\
\hline Striation machining parameters & \multicolumn{3}{|l|}{ Value } \\
\hline Traverse velocity, $\mathrm{vh}_{\mathrm{h}}(\mu \mathrm{m} / \mathrm{s})$ & \multicolumn{3}{|l|}{500} \\
\hline Approximate current density, $J_{n o z}\left(\mathrm{~A} / \mathrm{cm}^{2}\right)$ & \multicolumn{3}{|l|}{$20,100,300$} \\
\hline
\end{tabular}




\subsection{Materials preparation and characterisation methods}

A dual phase brass (Cu39Zn2Pb) was selected as the workpiece material in this study. This was selected as an adequate material in this study due to the high degree of control over the microstructure during heat treatment, and well-researched anodic dissolution phenomena in this material system. All samples were generated from the same feedstock, of nominal composition outlined in Table 2, from which the weighted alloy electrochemical equivalent (ECE) was calculated, according to

$$
E C E=\frac{m_{a}}{F z}
$$

giving a range considering minimum and maximum concentrations, where atomic mass, $m_{a}$, Faraday's constant, $F$, and valence, $z$. For simplification, each element is assumed to dissolve into one valence state, where $z$ : $\mathrm{Cu}$ (II), Zn (II), Pb (II). Difference in corrosion resistance between $\mathrm{Cu}$ and $\mathrm{Zn}$ components was appraised considering standard oxidation potential $\left(\mathrm{Cu} \rightarrow \mathrm{Cu}^{2+}+2 \mathrm{e}^{-}=-0.340 \mathrm{~V} ; \mathrm{Zn} \rightarrow \mathrm{Zn}^{2+}+2 \mathrm{e}^{-}=0.763 \mathrm{~V}\right.$, vs SHE), taken from Lange (1999), indicating that $\mathrm{Zn}$ is significantly easier to oxidise than $\mathrm{Cu}$.

Table 2: Nominal material composition and calculated weighted electrochemical equivalent at minimum and maximum compositions.

\begin{tabular}{|l|l|l|l|l|l|l|}
\hline & Cu (wt. \%) & Cu (at. \%) & Zn & Pb (wt. \%) & Pb (at. \%) & ECE (mg/C) \\
\hline Min. & 58 & 59.3 & Balance & 1.5 & 0.5 & 0.337 \\
\cline { 1 - 4 } Max. & 60 & 61.7 & & 2.5 & 0.8 & 0.339 \\
& & & & & & \\
\hline
\end{tabular}

The as-received microstructure, shown in the optical micrograph in Figure 1a, is typical of rolled brass. Separate sample pieces were cut from this feedstock and heat-treated 
for 60 minutes over a range of temperatures $\left(450,550,650,700^{\circ} \mathrm{C}\right)$, before being removed and water quenched. In addition, another sample was processed in an identical fashion at $800^{\circ} \mathrm{C}$, and subsequently tempered for 30 minutes $\left(500^{\circ} \mathrm{C}\right)$, generating a finer microstructure than the as-received condition. Samples of each precondition were etched using acidified ferric chloride solution, as detailed in Walker and Tarn (1991), for microstructural inspection. Optical micrographs were acquired using a focus-variation (FV) microscope (Alicona G5 Infinitefocus, 20x objective), and resulting microstructures are shown in Figure $1 \mathrm{~b}$-f respectively. Predictable increases in grain size with annealing temperature are observed, described by Humphreys (2004), as well as changes in the resulting phase ratio, with a-phase (lighter colour) concentration decreasing as the annealing temperature is increased.

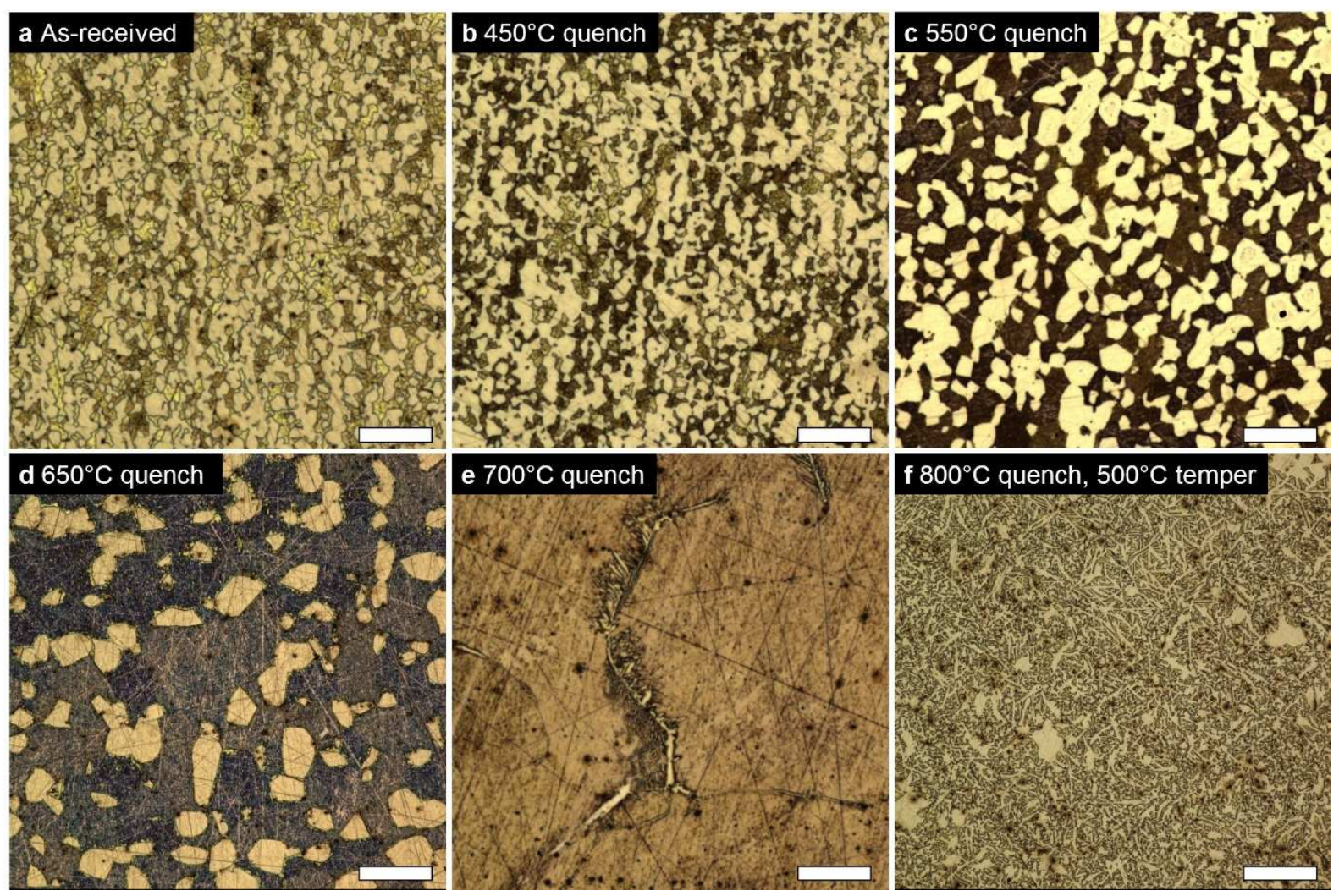

Figure 1: Etched microstructures of the brass samples by precondition. An increase in grain size is observed with increasing annealing temperature, as well as a decrease in apparent $\alpha$ phase. All scale bars $50 \mu \mathrm{m}$. 
The component phase ratio, and grain size, were quantified according to the methodology in Figure 2. From an etched surface, shown in the optical micrograph (see Figure 2a), energy dispersive X-ray spectroscopy (EDS) was performed in order to characterise the phase compositions ( $\mathrm{Cu}$ and $\mathrm{Zn}$ only) in the respective samples (see Figure 2b). In this study, the contribution to the machined surface from $\mathrm{Pb}$ was ignored as this phase is insoluble in brass and is known to form small $(1-2 \mu \mathrm{m})$ discrete globular particles. Due to the large atomic mass, the volume fraction of this element is relatively small, shown in a similar leaded brass by García et al. (2010). EDS was undertaken using an Oxford Instruments X-Max 80 detector $(20 \mathrm{kV}$ beam, $60 \mathrm{~s}$ acquisition time, minimum 6 spectra per phase per precondition). Binary images were generated from optical micrographs after thresholding in ImageJ, (see Figure 2c), where $\alpha$-phase grains appear white (grayscale, 0 ), and regions of $\beta$-phase black (grayscale, 255). Appraisal of the number of pixels corresponding to each phase in the whole binary image allows for estimation of the phase ratio, shown in Figure $2 \mathrm{~d}$. From the binary image, line profiles can be acquired (see Figure 2e), from which the grain sizes were quantified, shown in Figure 2f. Profiles were taken at four different orientations for each micrograph in order to negate directional effects associated with rolled materials. This method counts grains through the identification of grain boundaries, and therefore is less accurate when appraising the size of interconnected grain structuring. This is exemplified for the basket-weave microstructure, shown in Figure 1f, compared with samples of discrete grains, as it cannot characterise the interconnected nature of the basket-weave matrix. However, it instead gives an approximation of the $\alpha$-phase lath diameter in the subject. Applying Equation 1, the ECE of given phases can be calculated through weighting of the pure material ECE, applying the respective phase compositions of $\mathrm{Cu}$ and $\mathrm{Zn}$ (after conversion to atomic 
$\%$ ), observed experimentally by EDS (see Figure $2 b$ ). Thus, $\beta$-phase ECE is always greater than $\alpha$-phase ECE (ECE for as-received precondition: $\alpha=0.3329 \mathrm{mg} / \mathrm{C}, \beta=$ $0.3335 \mathrm{mg} / \mathrm{C})$.
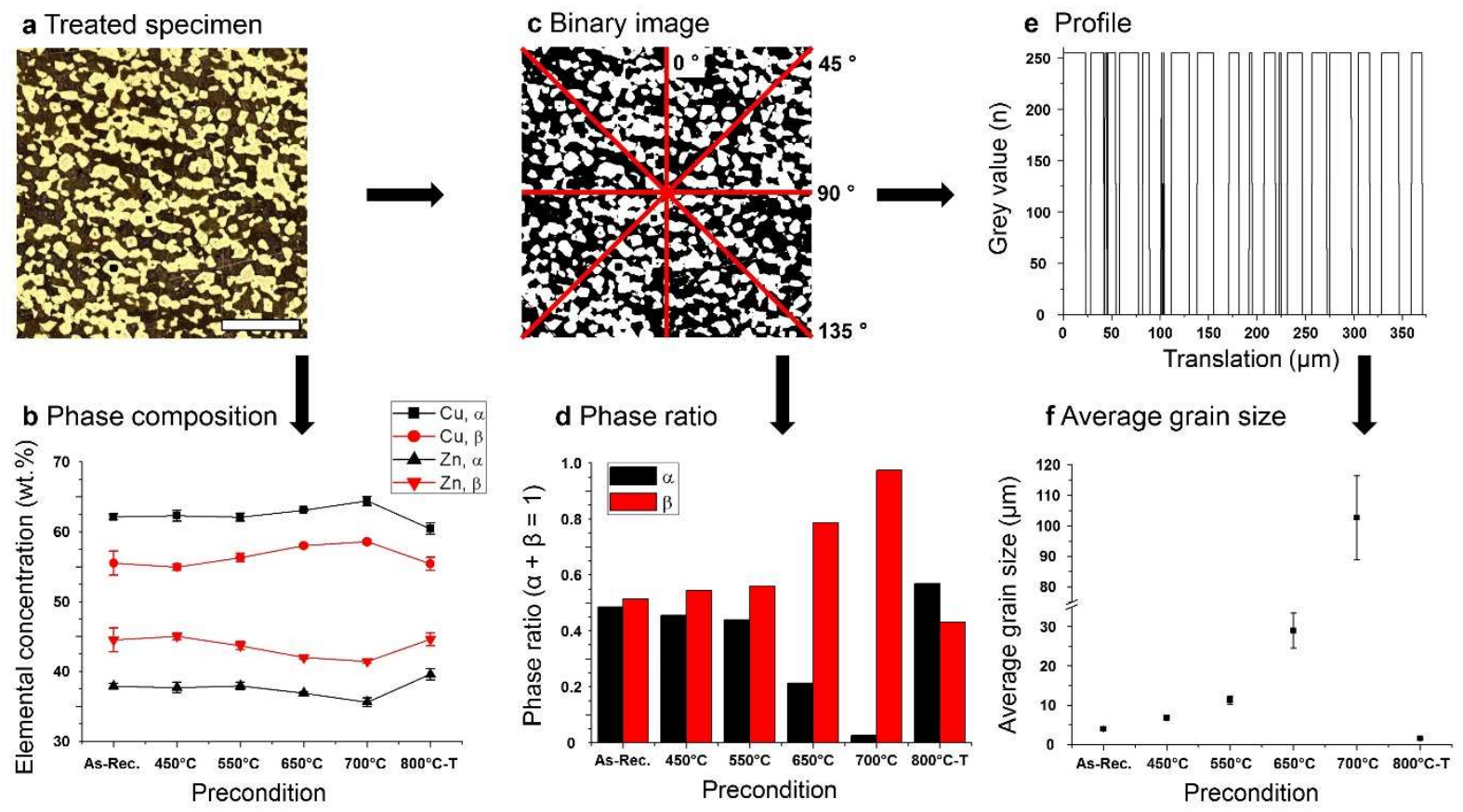

Figure 2: Effect of pre-treatment on microstructure and phase composition. a) Optical micrograph of etched surfaces. b) EDS phase compositions at each precondition. c) Binary image from optical micrograph, from which $d$ ) the phase ratio $(\alpha / \beta)$ can be obtained through pixel counting, $\alpha$-phase white and $\beta$-phase black. e) From the binary image, greyscale profiles can be acquired at different orientations, where each step $(0-255)$ corresponds to a grain boundary. f) grain boundary counting per length, at multiple orientations, allows average grain size approximation through the linear intercept method. Error bars are the standard deviation from the mean, calculated from the grain sizes returned from at least four profile orientations in each material. Optical scale bar $100 \mu \mathrm{m}$.

Post-machined topographic characterisation was undertaken using the aforementioned FV microscope (100x objective). Single exposures (160x160 $\mu \mathrm{m}$ field of view) were acquired from the basal area (exposed to the highest current density) of each feature and the surfaces were subjected to form removal ( $\left(2^{\text {nd }}\right.$ degree polynomial), 
and waviness filtering ( $80 \mu \mathrm{m}$ nesting index), in order to output a surface map, from which the areal height parameters: Sa and Sq, were extracted, following Leach (2011). The volume parameters $(V m p, V m c, V v c, V v v)$ were also extracted from the processed surfaces to obtain volume distribution information, with bearing ratios set throughout analysis $(p=10 \%, q=80 \%)$. The topography analysis routine is shown in Figure 3 . Material removal was characterised by measuring the pit volumes (FV microscope, $20 x$ objective), and the mass was inferred through consideration of the material density $\left(8.40 \mathrm{~g} / \mathrm{cm}^{3}\right)$ for this brass, taken from Nunes et al. (1979). All of these steps were undertaken using MountainsMap software. Scanning electron microscopy (SEM) was undertaken using a Philips XL-30 microscope. EDS analysis of sectioned sample pieces was undertaken using the aforementioned system, across a line-scan $(0.75 \mu \mathrm{m}$ inter-acquisition spacing, acquisition parameters as above).

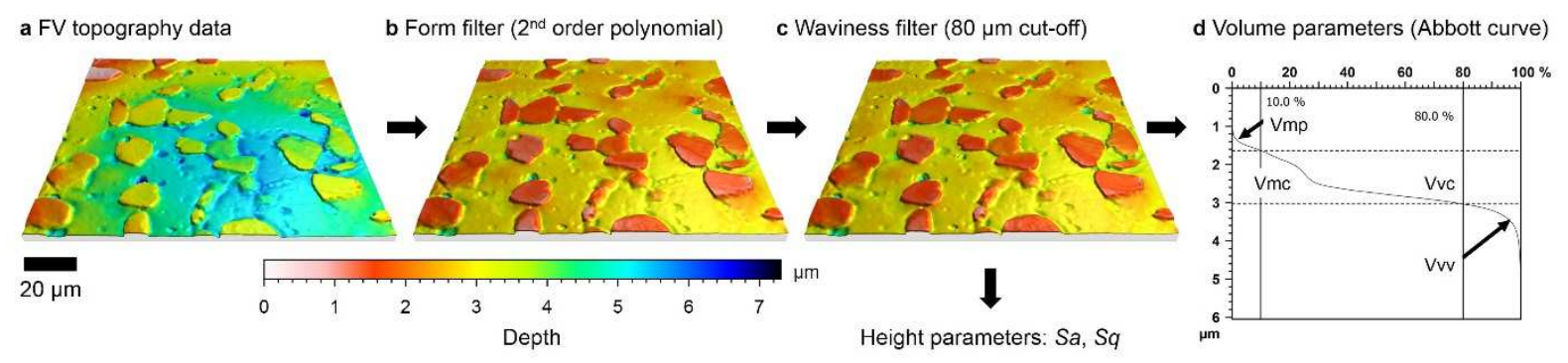

Figure 3: Topographical analysis routine in this investigation. 


\subsection{Results and discussion}

\subsection{Topography}

The workpieces were subjected to identical EJP point processing treatments, where the parameters are outlined in Table 1. A key characteristic of the brass surfaces appraised here, and indeed of multi-phase materials in general when processing with electrochemical techniques, is the preferential machining of one phase relative to another. In this case, the a-phase, which has a marginally lower electrochemical equivalent (ECE, see methodology) and significantly greater corrosion resistance, considering standard oxidation potentials (see methodology), is retained at the surface, while the $\beta$-phase is removed preferentially. Evidence for this is shown in Figure 4, for the preconditions machined at identical EJP treatments $\left(100 \mathrm{~A} / \mathrm{cm}^{2}\right)$. In each surface reconstruction, the $\alpha$-phase protrudes from the surface, expected as a result of the lower ECE and higher corrosion resistance, except for the predominantly single-phase workpiece $\left(700^{\circ} \mathrm{C}\right.$, see Figure $\left.4 \mathrm{j}\right)$. Even in this workpiece however, retained a-phase present at the grain boundaries, is shown to protrude in the accompanying SE micrograph (see Figure 4i). The SE micrographs and accompanying reconstructions show that the observable surface is closely related to the underlying material microstructure, and larger prior a-grain sizes are retained at the surface after machining.

As the material microstructures tend towards single-phase $\left(550,650,700^{\circ} \mathrm{C}\right.$ conditions) and the number of $\beta / \beta$ grain boundaries increases (as a proportion of the total boundary length), intergranular attack of $\beta / \beta$ grain boundary areas becomes clear. This manifests as multiple hemispherical (isotropic) micro-pits along the length 
of the grain boundary. This is likely the result of the lower activation energy required for the liberation of a given metal atom as the long-range order of the crystalline lattice is disrupted in this area. The more electrochemically inert nature of the $\alpha$-phase appears to prevent these isotropic pitting events from occurring at dissimilar $\alpha / \beta$ grain boundaries. Material removal instead occurs around a given $\alpha$-phase grain in a homogenous manner, resulting in undermining. This is due to a current-focussing effect, resulting in highly localised current density increases at the edges of $\alpha$-phase grains. This effect has been simulated for inert ceramic-type phases by HackertOschätzchen et al. (2016) and leads to the depression of the inter-boundary zone on the $\beta$-phase side, which is easily observed in the $650^{\circ} \mathrm{C}$ annealed condition (see Figure 4h). 


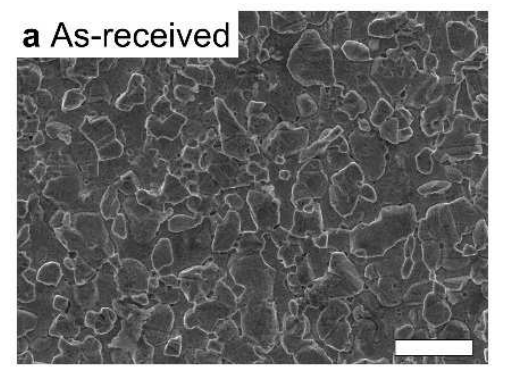

b
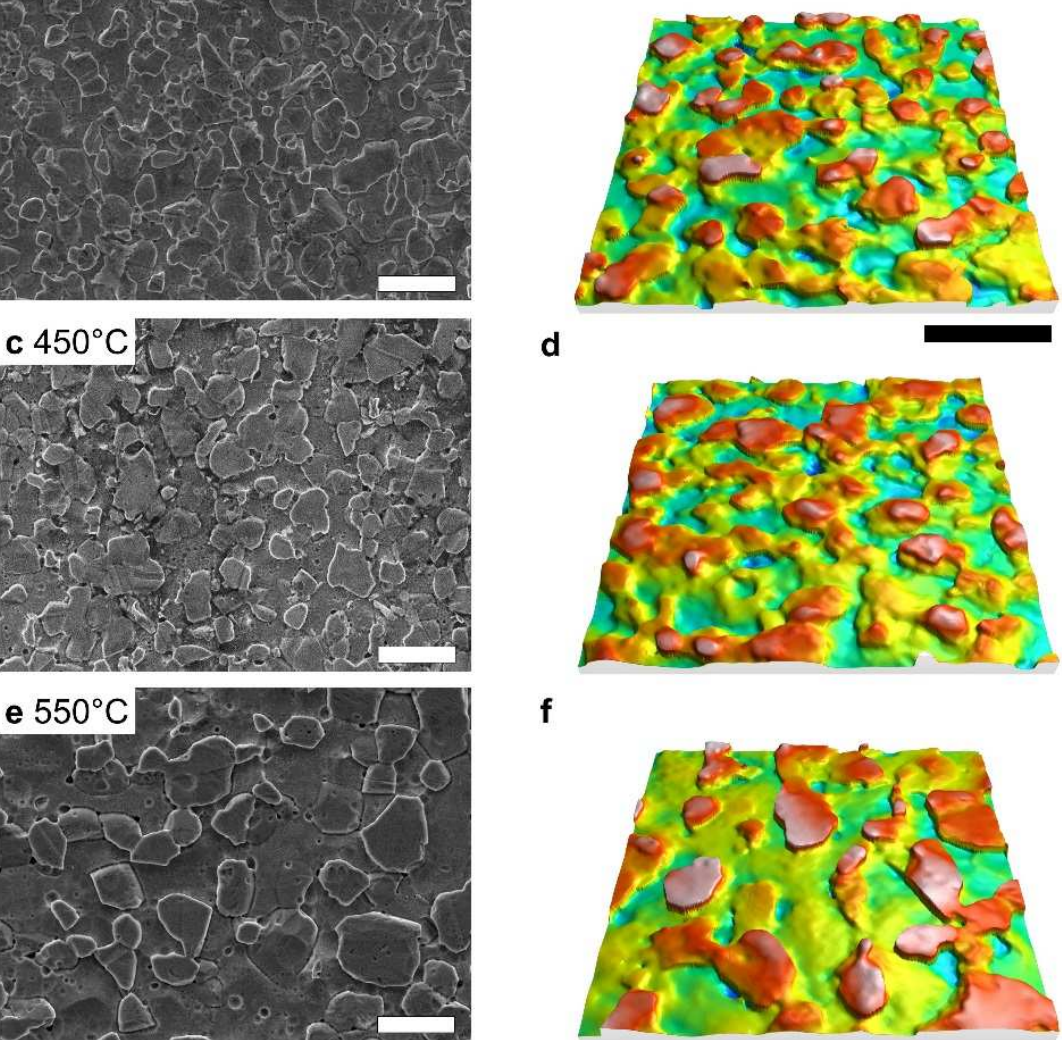

f
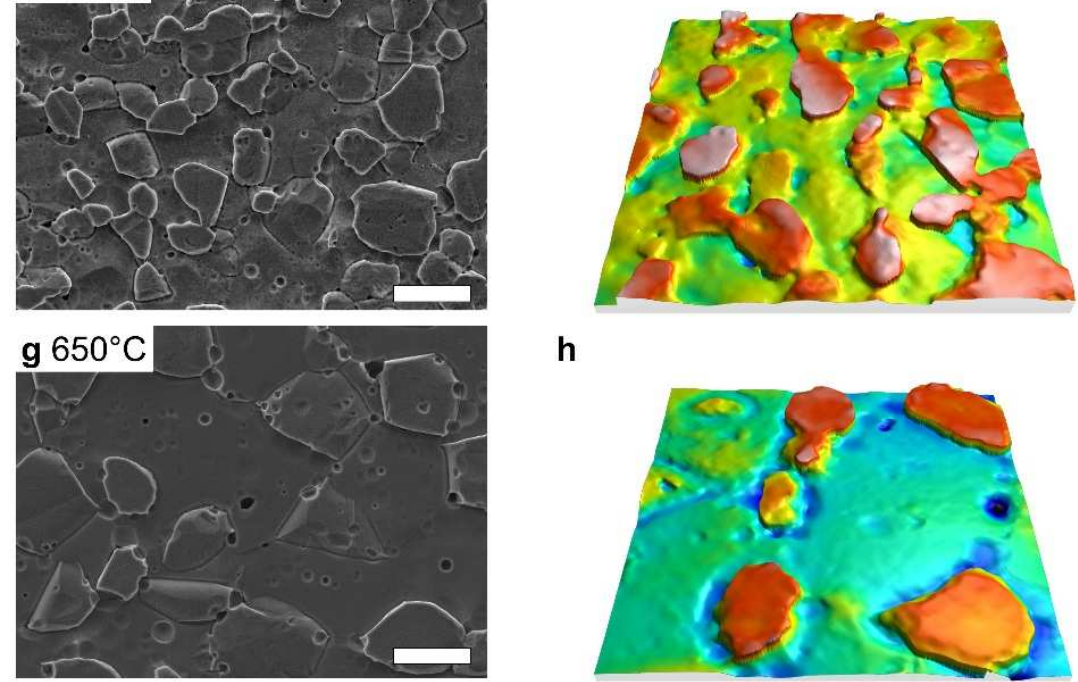

h
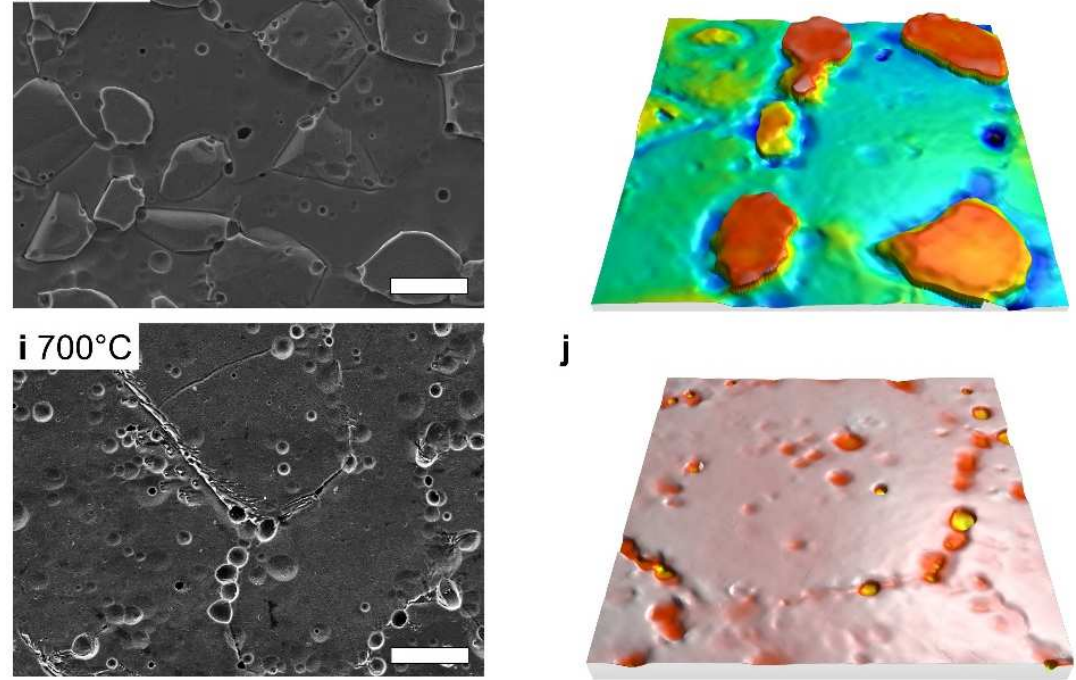

j
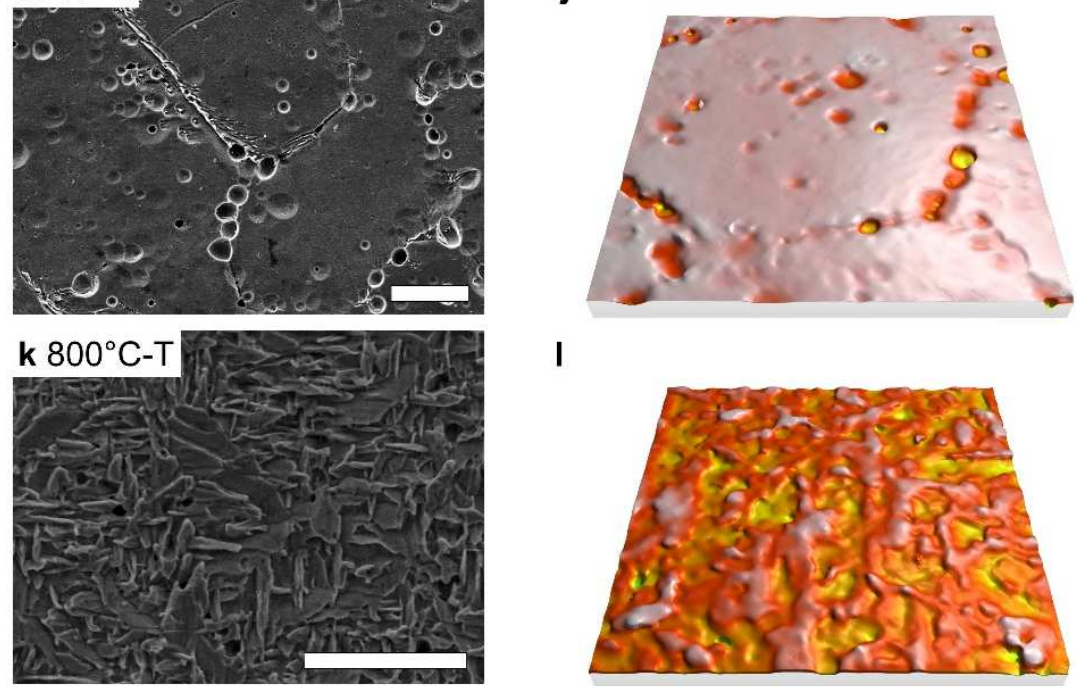

I
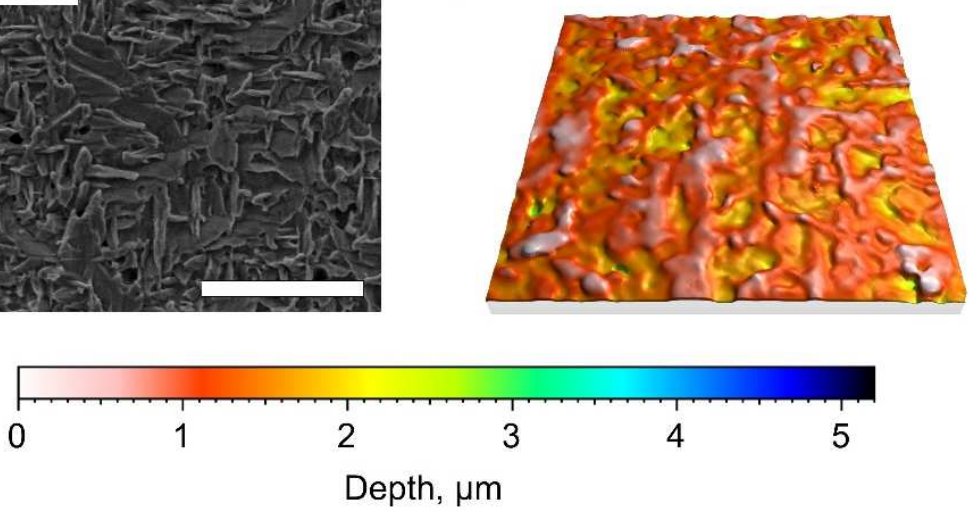
Figure 4: Surfaces resulting from equivalent EJP operations $\left(100 \mathrm{~A} / \mathrm{cm}^{2}\right)$ on various workpieces. SE micrographs (2500x magnification), a, c, e, g, i, k), which show the protrusion of $\alpha$-phase grains from the surface after electrochemical treatment in all cases, including i, where fine $\alpha$-phase structures can be observed in boundary areas. Surface reconstructions (100x magnification, $75 \times 75 \mu \mathrm{m}$ field of view), b, d, f, h, j, I) showing that the relative protrusion of surface phases can be quantified, respective to the colour scale. Scale bars $20 \mu \mathrm{m}$.

Topographies were quantified using FV microscopic data (100x objective), using the metrics of areal arithmetic mean height, $S a$, and root mean square height, $S q$, to describe the surface amplitudes. In both datasets, shown in Figure 5, the surfaces for all of the workpieces appear to follow similar power-law trends with applied energy, although the magnitude of the height changes with precondition in some cases. For the as-received, $450^{\circ} \mathrm{C}$, and $550^{\circ} \mathrm{C}$ annealed samples, no difference in height beyond the error range is detected for current densities $>50 \mathrm{~A} / \mathrm{cm}^{2}$, although there is some variation at the lowest current density tested $\left(20 \mathrm{~A} / \mathrm{cm}^{2}\right)$. The smallest heights, corresponding to the smoothest surfaces were achieved with the $700^{\circ} \mathrm{C}$ annealed workpiece, a result of the predominantly single-phase nature of the material, which leads to a significant reduction in the amount of protruding a-phase.

From this data alone, it is not possible to determine the impact that grain size has on the surface amplitude $(S a, S q)$ of pits, as the phase fractions of $\alpha$ - and $\beta$ - have also been varied in the pre-treatment. Where the phase ratio tends towards one phase, lower roughness is observed, a consequence of the reduction in the influence of relative machining rates on the resulting surface. However, from the data presented it is clear that grain size does have some effect on the roughness. For example, the 
$800^{\circ} \mathrm{C}$-tempered condition, having the smallest grain size (approximately $1.5 \mu \mathrm{m}$ ) and a similar phase ratio to the as-received, $450^{\circ} \mathrm{C}$, and $550^{\circ} \mathrm{C}$ samples results in lower surface roughness after equivalent processing.

a Arithmetic mean height, Sa

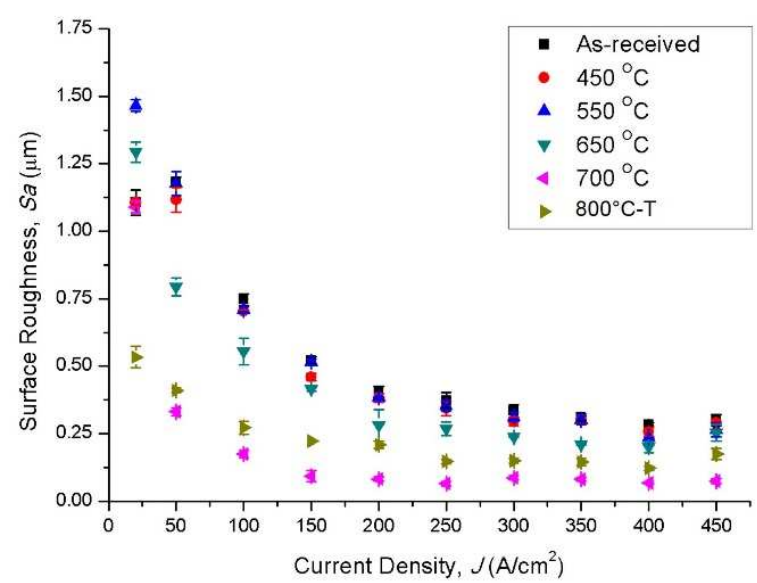

b Root mean square height, $S q$

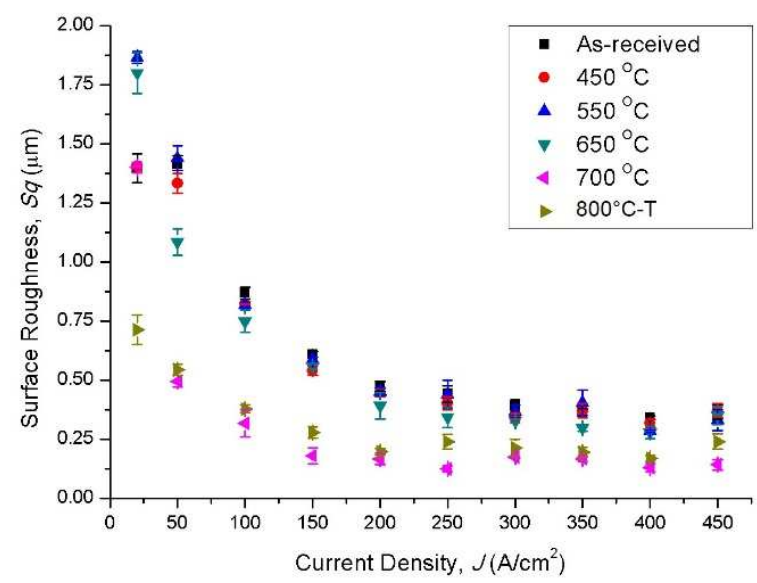

Figure 5: Surface height analysis for pit centres. a) Sa, b) Sq. Error bars are the standard deviation from the mean for the central pit area roughness of the three machined pits at each data point.

Surfaces were subsequently appraised with respect to their volume parameters; peak material volume $(V m p)$, core material volume $(V m c)$, core void volume $(V v c)$, and valley void volume $(V v v)$, in order to characterise the distribution of surface volume with respect to current density and material precondition. Extracted data are shown in Figure 6. For all volume parameters, an inverse relationship is observed with regard to current density, indicating a smoother surface due to electropolishing effects, correlating well with areal height analyses (see Figure 5). At low current densities, the $650^{\circ} \mathrm{C}$ annealed condition is shown to have the greatest $V m p$. This is likely to be the result of the largest $\alpha$-phase grain size, which protrudes from the surface after EJP. Conversely, the tempered sample (smallest $\alpha$-phase grain size) and the $700^{\circ} \mathrm{C}$ annealed condition (predominantly $\beta$-phase), show the lowest $V m p$. In terms of valley 
void volume, the $700^{\circ} \mathrm{C}$ annealed condition shows the most, correlating well with Figure $4 \mathrm{j}$, representing a quantification of the prior-observed intergranular attack of homogenous $\beta / \beta$ boundaries in the predominantly single-phase material. For all surfaces except the $700^{\circ} \mathrm{C}$ annealed, and $800^{\circ} \mathrm{C}$-tempered conditions, $V m c$ data is lower than $V v c$ (see Figure $6 \mathrm{~b}$ and $\mathrm{c}$ ), which indicates a significant fraction of the total surface volume being empty space. This is expected as these workpieces are rich in $\beta$-phase, which preferentially machines to leave a topography dominated by protruding a-phase grains. The difference between $V m c$ and $V v c$ is reduced somewhat, as current density is increased, demonstrating flatter surface topographies, likely to result from a tendency towards a more equal removal of $\alpha$ - and $\beta$-phases as electropolishing effects increase.
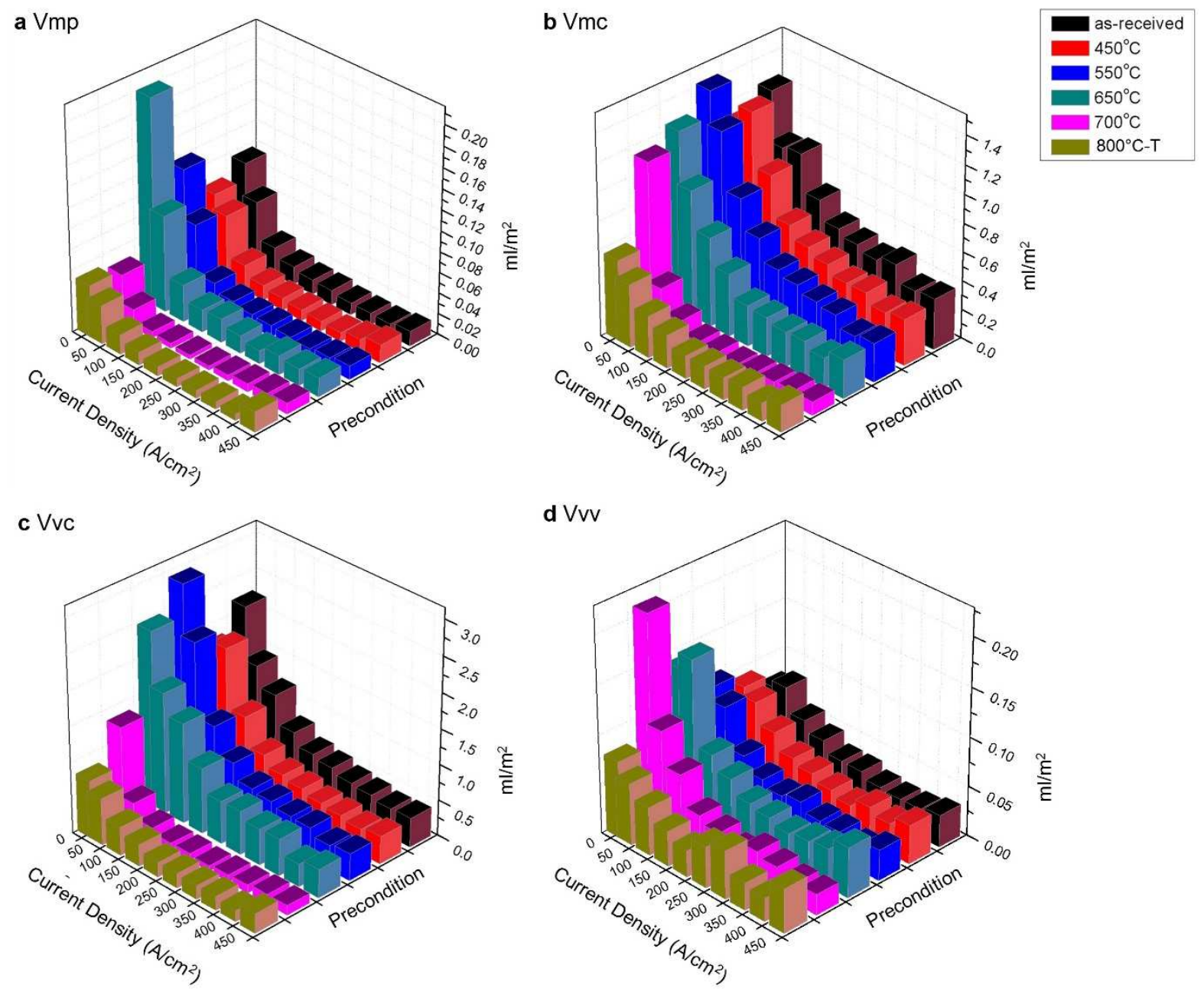
Figure 6: Volume parameters from the surfaces of pits machined in the different brass microstructures. a) $V m p$ (peak material), b) $V m c$ (core material), c) $V v c$ (core void), d) $V v c$ (valley void).

Surface amplitudes observed in point machining are not representative of the conventional vector approach to EJP, which is most commonly practised. In this case, the electrolyte jet is translated across the workpiece as part of a two-dimensional toolpath, whereby the current density distribution lead-in and lead-out are lower than the central processing regime in the jet. The latter affects the resulting topography, lowering the effective surface defining current density. This implies that microstructural differences that cause insignificant differences in point processing are likely to affect the topography across a broader $J_{\text {noz }}$ range, in vector processing. To appraise the microstructural effects in an environment more similar to a conventional electrochemical jet process, striations were machined in identically treated samples at equivalent feed rate and at three current densities $\left(J_{\text {noz }}=20,100,300 \mathrm{~A} / \mathrm{cm}^{2}\right)$, detailed in Table 1.

At all tested current densities, there is a clear increase in surface amplitude with grain size, up to the $550^{\circ} \mathrm{C}$ annealed condition, at which point surface amplitude decreases, even as grain size is significantly increased (see Figure 7a). This results from the increasing phase-fraction of the dominant $\beta$-phase, leading to a machined surface more typical of a single-phase material. For this material, there is a shift in the surfacedefining mechanism becoming less dependent on the grain size, and more dependent on the phase ratio. This effect is shown to be independent of current density within the ranges tested. 
The low surface amplitudes (in comparison to point machining) attained at low current density $\left(J_{n o z}=20 \mathrm{~A} / \mathrm{cm}^{2}\right)$ appear uncharacteristic. Here, a fraction of the measured surface is comprised of a-phase grains from the original sample surface, subjected to the preparatory surface polishing routine, indicating that in this case, the amplitude is defined by the material removal as well as a microstructural characteristic. In contrast, at higher current densities, where all of the prior surface was removed in the process, larger surface amplitudes are observed. Despite this, in vector processing, the dependence on current density of the surface amplitude is reduced in comparison with point machining and microstructural effects dominate the topography. This is due to an equilibration effect, whereby the final current density experienced by a given point of the machined surface is lower (lead-out of the $J$ distribution) than that experienced by the central region of a machined pit. Therefore the surfaces are defined by more similar finishing current densities than implied by the supplied current densities $\left(J_{n o z}\right)$. 
a Surface amplitude

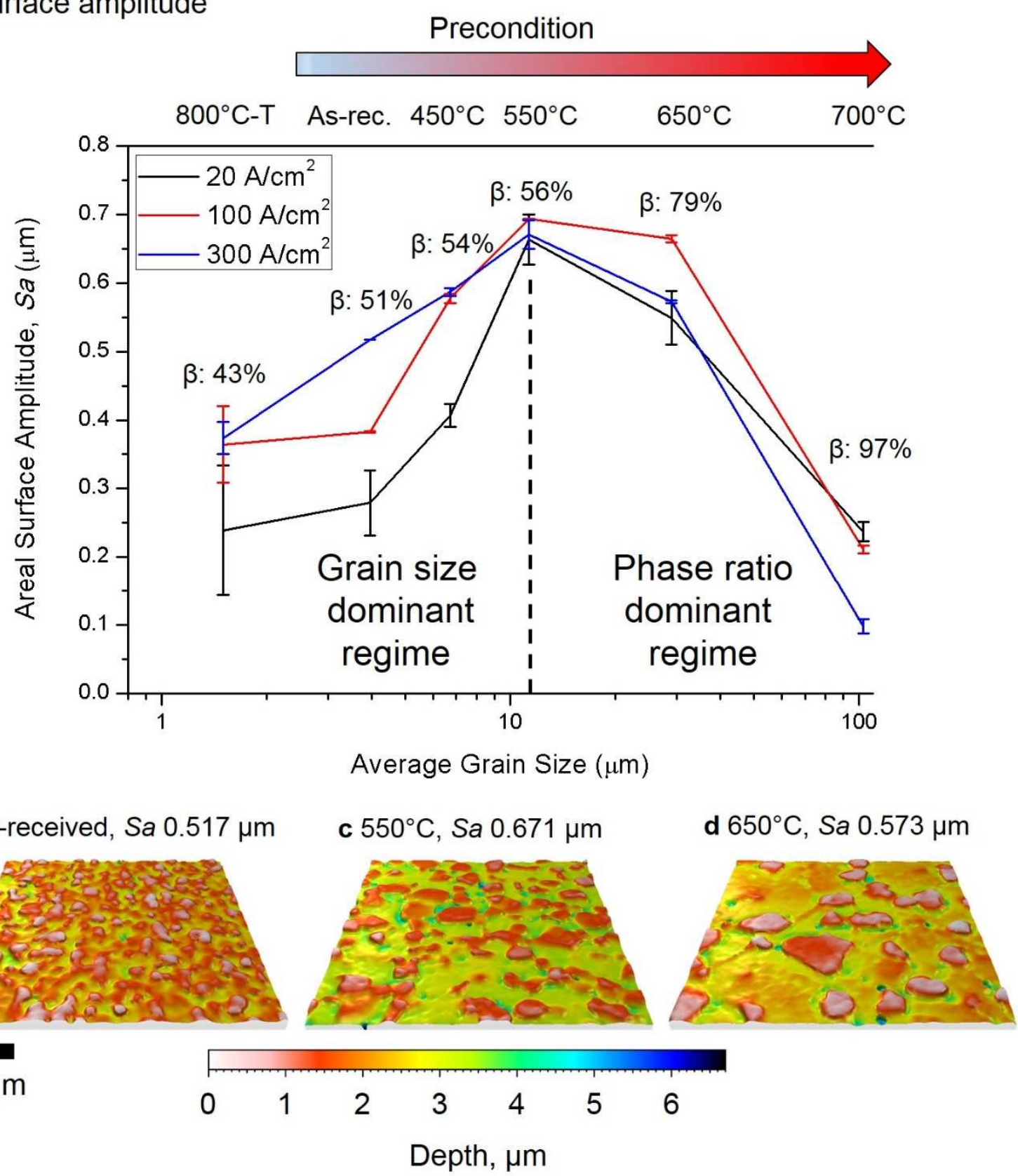

Figure 7: a) Surface amplitude, Sa, of striations machined at three current densities $\left(J_{\text {noz }}=\right.$ $20,100,300 \mathrm{~A} / \mathrm{cm}^{2}$ ) in the different material preconditions (note log scale). Topographic reconstructions from samples machined at high current density $\left(300 \mathrm{~A} / \mathrm{cm}^{2}\right)$, where surface amplitude increases with grain size from b) the as-received material to c), the $550^{\circ} \mathrm{C}$ precondition, and then decreases for preconditions beyond, as the surface volume of protruding a-phase decreases, d) shown for the $650^{\circ} \mathrm{C}$ precondition. 


\subsection{Relative phase machinability}

The apparent shift in dominant surface-defining mechanism from grain size to phase ratio in this material can be understood through consideration of the relative phase machinability. This was appraised for the different preconditions, through the quantification of step heights, between $\alpha$ and $\beta$-phase of the point-machined sample set. A larger step height is indicative of greater relative machinability of a given phase. In Figure 8a, difference in machinability is shown to decrease as the current density is increased, from low $J(\alpha<<\beta)$, to high $J(\alpha<\beta)$. This supports the prior appraisal of pit surface roughness, Sa, Sq, decreasing as the current density is increased (see Figure $5 a$ and $b)$, which is indicative of the difference in machinability reducing as current density is increased.

In addition, there appears to be a correlation with grain size at low current density, where the difference in machinability is greatest. Referring back to Figure 7 (vector processing), step height increases with grain size, (defining the first regime), increasing roughness. However, the number of discrete steps decreases as the phase ratio becomes more polarised, which leads to a reduction in surface amplitude (defining the second regime). The correlation between grain size and step height is exemplified in the extracted profiles at low (see Figure 8b) current density (for the two grain size extremes), where step heights are demonstrably greater with increasing grain size. At higher current density (see Figure 8c), grain size has a lesser effect on relative machinability, and profiles are approximately equivalent in step height. At higher current densities, where electropolishing mechanisms dominate in EJP, and the relative machinability tends towards the electrochemical equivalent of the phases, 
the amplitude of the machined profiles appears to be more equivalent, although the surface frequency, which is governed by the grain size, is different. This is supported by the data shown in Figure 8a, demonstrating that the average step height is smaller than the average grain size.

Grain size itself is not thought to make a significant difference to the relative machinability of given phases, as this is dependent on $J$ and the composition of both the material and the electrolyte, however changes in grain size affect the step height between the two phases. The difference in step height with grain size, at $J<50 \mathrm{~A} / \mathrm{cm}^{2}$, is proposed to be the result of an anchoring effect, wherein larger $\alpha$-phase grains are retained in the workpiece for longer as the surface evolves into the material. Larger grains therefore allow greater protrusion.

Where the relative machinability of $\alpha<<\beta$, (low current density), step height should tend towards $\alpha$-phase grain size, however, the quantified result is smaller, with a large data spread. The spread can be partially explained by the stochastic distribution of $\alpha$-phase grains within the material (see Figure 8d), where a discrete step height depends on the position of a given $\alpha$-grain relative to the dissolution front (see Figure $8 \mathrm{e}$ ). In addition, the step height will reduce from the average grain size, especially for a largegrained sample. As the dissolution front evolves into the workpiece during dissolution (increasing height difference), exposed a-phase grains will be subject to electrochemical attack regardless of the preferential machinability of the $\beta$-phase, as i) they will act as current-focussing features and ii) the a-phase surface area subject to attack will increase (see Figure 8f). 


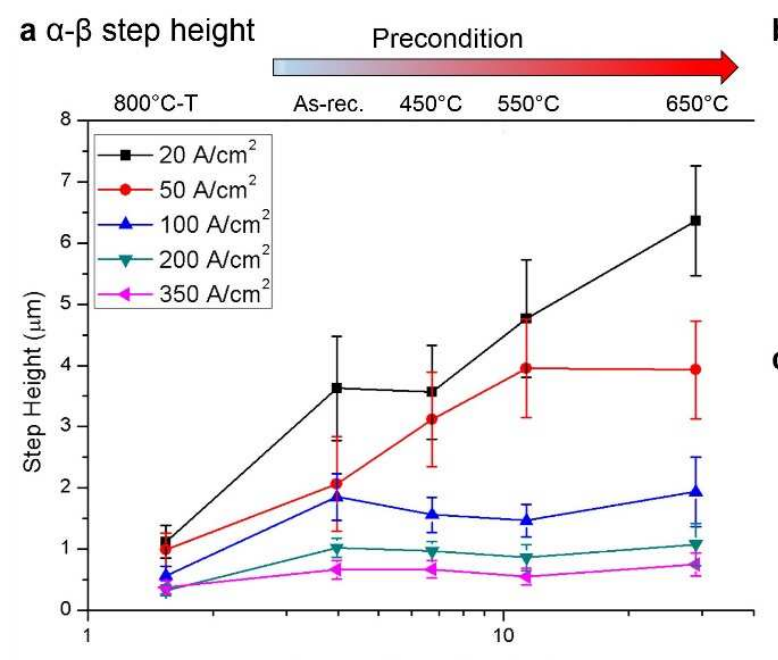

b Low $J$
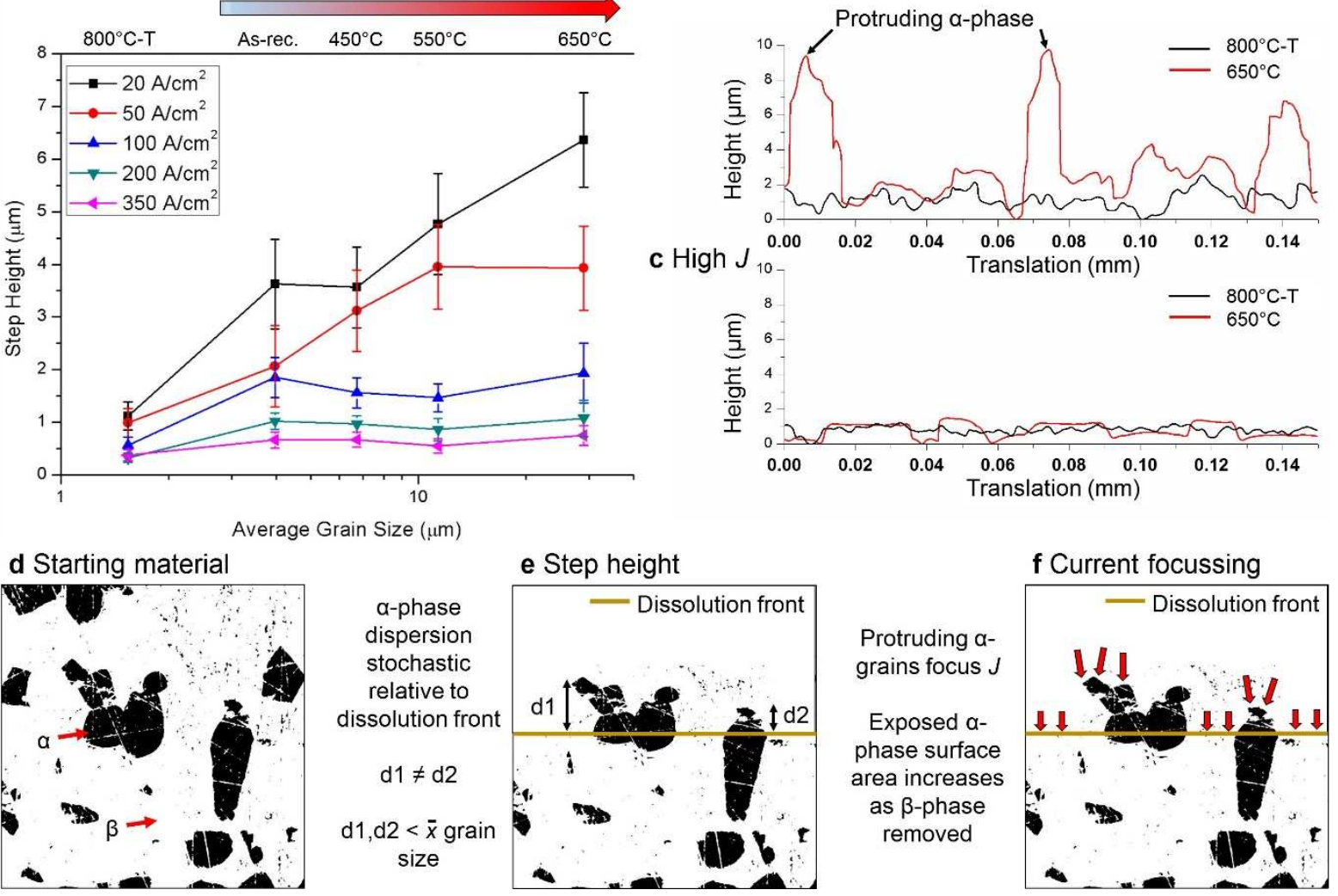

Figure 8: Step height measured between $\alpha$-phase and preferentially machined $\beta$-phase across a range of current densities $\left(20-350 \mathrm{~A} / \mathrm{cm}^{2}\right)$, for the material preconditions. Step height increases with grain size, evidenced in the exemplar extracted profiles from the extreme grain sizes in this study at low and high current density, b) and c) respectively. d) Schema viewed in cross section ( $\alpha$-phase black; $\beta$-phase white), step height is less than average grain size due to e) the stochastic distribution of $\alpha$-phase within the material and relative to the dissolution front and f) current focussing effects, which limit protrusion. 


\subsection{Near-surface chemistry}

In addition to difference in machinability between the two phases, compositional changes are observed at the near-surface due to the different oxidation potentials of the elements present. As the more corrodible component of brass, considering oxidation potentials, it is more energetically favourable to liberate $\mathrm{Zn}$ from the surface compared with $\mathrm{Cu}$, thus the relative surface concentration of relatively corrosion resistant $\mathrm{Cu}$ increases. This was appraised for sectioned striations across current densities $\left(20,100\right.$ and $\left.300 \mathrm{~A} / \mathrm{cm}^{2}\right)$ and is shown in Figure 9. Zn concentration is depleted at the near-surface layer $<1.5 \mu \mathrm{m}$ in depth, especially at the lowest current density tested in this study. At $300 \mathrm{~A} / \mathrm{cm}^{2}$, surface concentration of $\mathrm{Zn}$ is more representative of the bulk material, indicating an increased rate (per charge passed) of $\mathrm{Cu}$ removal. This is largely thought to result from the surface dissolution rate, which is slow at $20 \mathrm{~A} / \mathrm{cm}^{2}(\leq 4 \mu \mathrm{m} / \mathrm{s}$ at jet centre; where the maximum surface dissolution rate is the ratio between the maximum pit depth and the machine time), when compared with a high current density of $300 \mathrm{~A} / \mathrm{cm}^{2}(\leq 65 \mu \mathrm{m} / \mathrm{s}$ at jet centre). When the rate of bulk material removal is low, the surface is exposed to the action of the electrolyte for a longer time period, thus selective removal between $\mathrm{Cu}$ and $\mathrm{Zn}$ is increased. This builds a Cu-rich layer over the $\beta$-phase as the $\mathrm{Zn}$ is preferentially leeched. Conversely, where the dissolution rate is greater, selective removal is significantly reduced, such that the surface composition is more representative of the bulk material (see Figure 9f and g). The Cu-rich build-up at low current densities is likely to limit the preferential removal of the $\beta$-phase as dissolution evolves. 

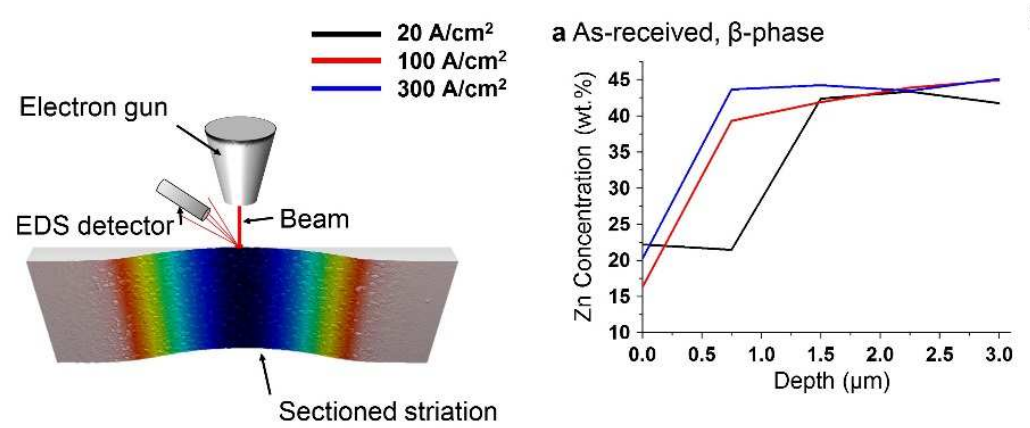

b $450^{\circ} \mathrm{C}, \beta$-phase
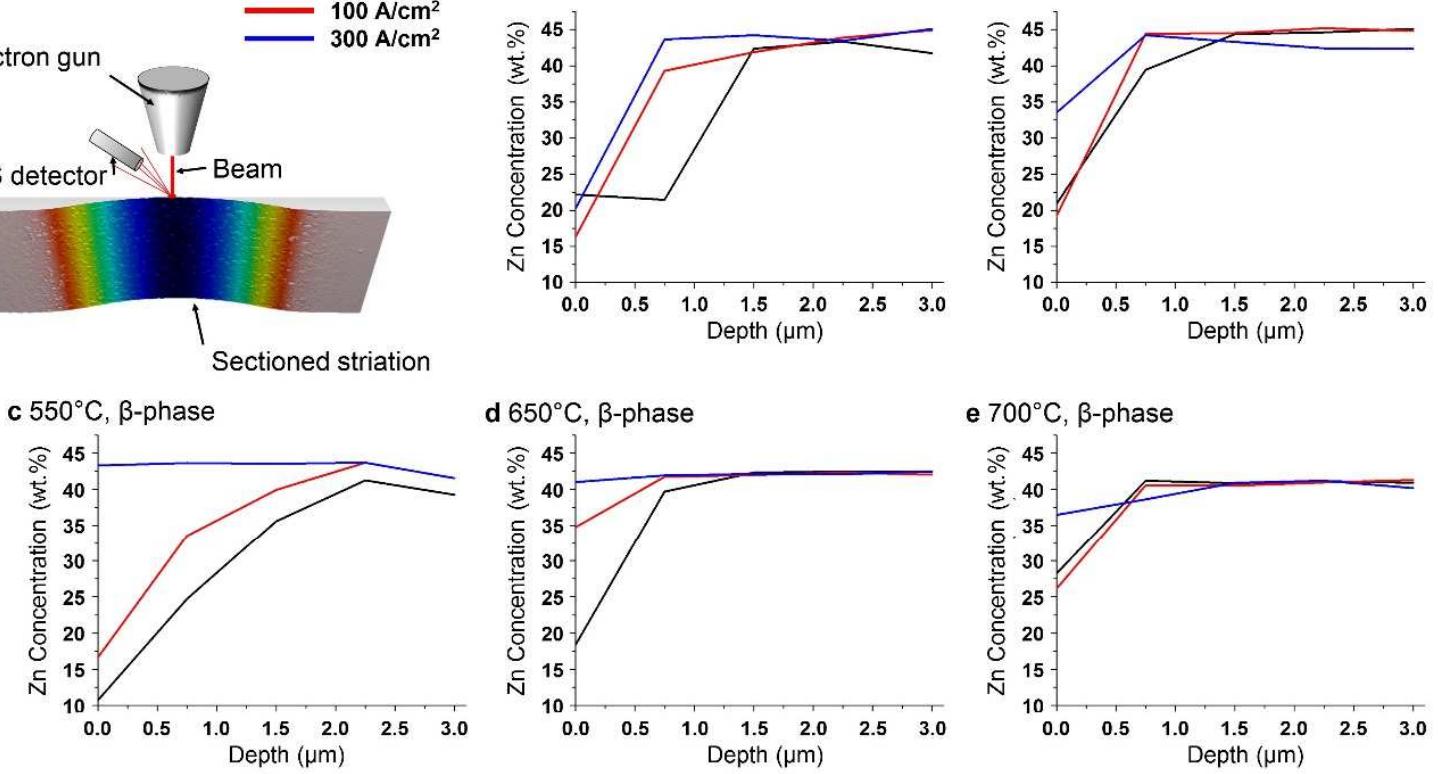

e $700^{\circ} \mathrm{C}, \beta$-phase

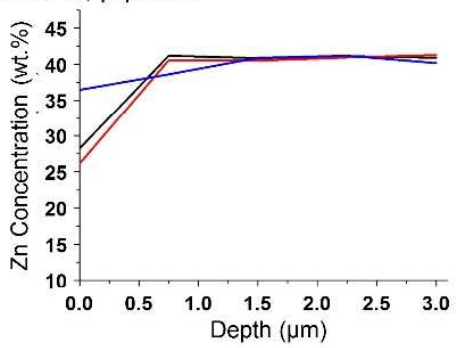

f Starting material

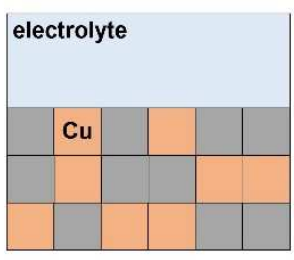

g Low current density, $20 \mathrm{~A} / \mathrm{cm}^{2}$

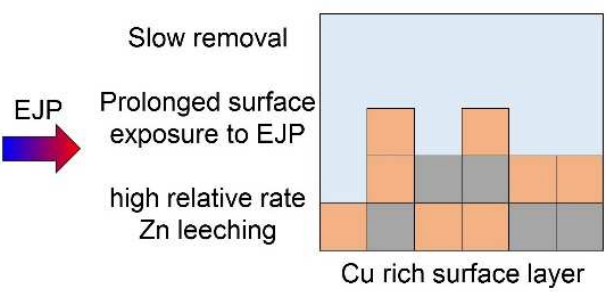

h High current density, $300 \mathrm{~A} / \mathrm{cm}^{2}$

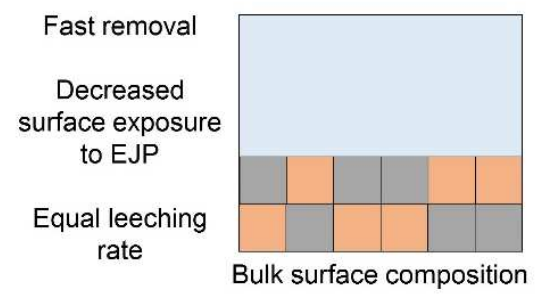

Figure 9: Spectral acquisition of $\beta$-phase $Z n$ concentration (wt. \%) of sectioned tracks $\left(J_{\text {noz }}=\right.$ 20, 100 and $300 \mathrm{~A} / \mathrm{cm}^{2}$ ), from EDS line-scans (6 points, $0.75 \mu \mathrm{m}$ intervals), where $\beta$-phase grains were selected at the track centres. Dezincification is observed in all cases, and is more pronounced at low current density. This is shown in the schema $f-h$ ), where the leeching rate is controlled by the current density. 


\subsection{Relative removal rates}

A consequence of poor a-phase machinability at low current density, is an erosive removal mechanism, whereby the inert phase is removed at a lower rate through anodic dissolution. Instead, as the surface evolves, $\alpha$-phase grains are undercut and subsequently washed away in the electrolyte jet. Supporting evidence for this effect is shown in the tinted SE micrograph of a sectioned surface in Figure 10a, processed at low current density, where a-phase grains are undermined by the preferential machining of the $\beta$-phase. In the reconstructed surface (see Figure 10b), this undermining effect can be appraised, complemented by the extracted profile (see Figure 10c), taken across points $\mathrm{i}-\mathrm{ii}$, which shows material removal exaggerated at the inter-phase grain boundary in this case, where the interface is up to $2 \mu \mathrm{m}$ deeper than the adjacent $\beta$-phase grains. Evidence for the erosion of $\alpha$-phase grains through undermining and wash-out mechanisms is shown in Figure $10 \mathrm{~d}$, of the $550^{\circ} \mathrm{C}$ annealed condition. Here, a recession where the width corresponds in magnitude to the average a-grain size in this material $(11.3 \mu \mathrm{m}$, see Figure 2$)$, can be seen in the central blue (deepest) area of the reconstruction. This is atypical of the surrounding surface and is likely to be the result of a-grain erosion. The accompanying SE micrograph of this area (see Figure 10e), shows a more irregular surface texture at the base of the recess when compared to the surrounding grains, indicative of a different removal mechanism. 

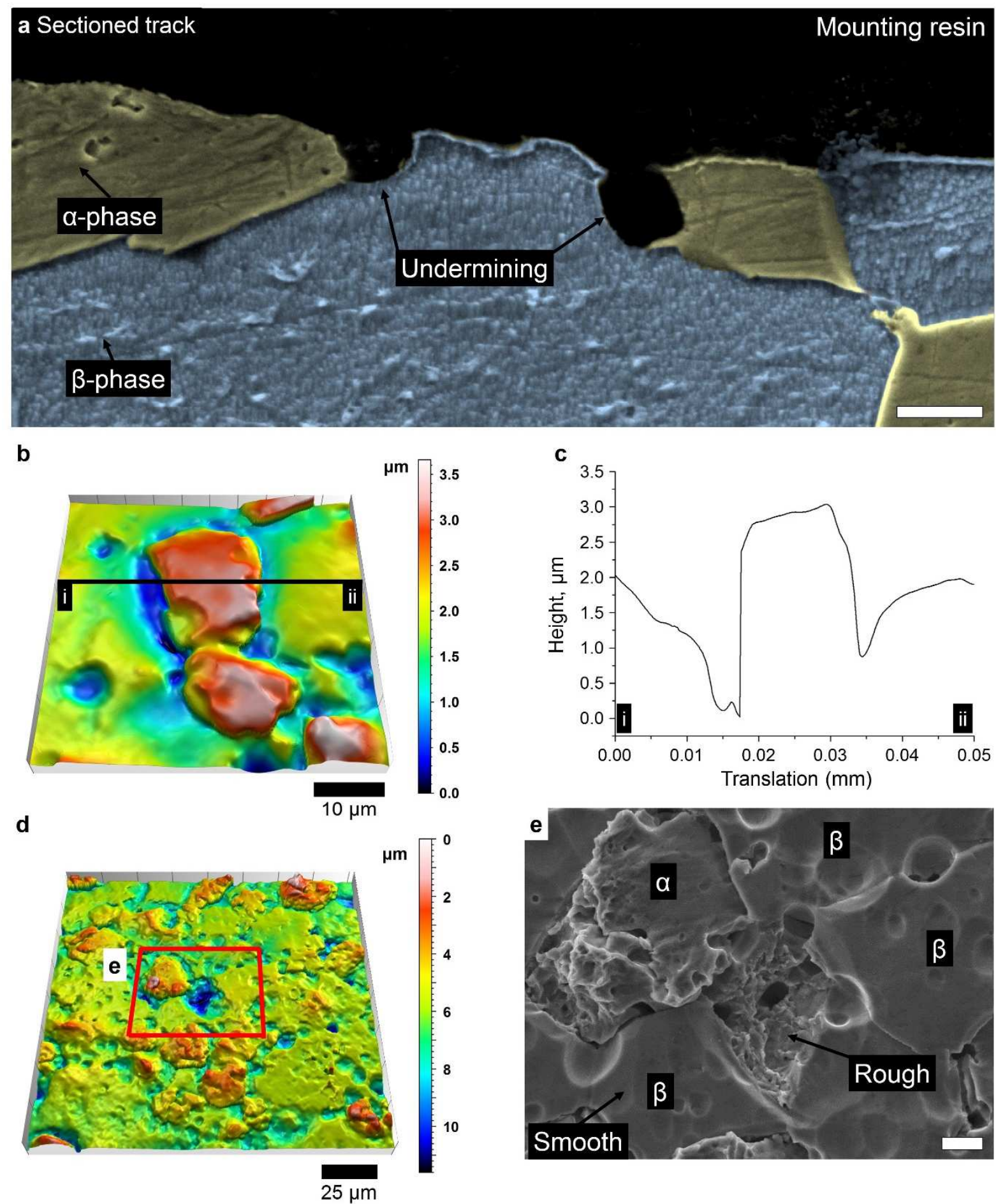

Figure 10: a) Tinted secondary electron micrograph ( $\alpha$-phase yellow; $\beta$-phase blue) of sectioned striation $\left(650^{\circ} \mathrm{C}, v_{h}=500 \mu \mathrm{m} / \mathrm{s}, 100 \mathrm{~A} / \mathrm{cm}^{2}\right)$, where $\alpha$-phase grains are undermined at the surface, b) surface reconstruction showing a-phase (protruding) undermining $\left(650^{\circ} \mathrm{C}, 100 \mathrm{~A} / \mathrm{cm}^{2}\right)$, c) profile extracted from surface. d) Recess from probable wash-out event, corresponding in magnitude to the average grain size in this material $\left(550^{\circ} \mathrm{C}, 50 \mathrm{~A} / \mathrm{cm}^{2}\right)$, atypical of surrounding surface, e) SE micrograph showing a different surface texture at the base of this recess. All scale bars are $5 \mu \mathrm{m}$. 
The influence of possible erosive effects on the removal efficiency, was appraised through measuring the removal mass per unit charge (actual ECE) for the pit samples, at each condition, as outlined in the methodology, and shown in Figure 11a. This was compared with the theoretical ECE for the feedstock material (lower - light, upper dark green, see Table 2).

For erosion complementing anodic dissolution, an inverse relationship would be expected between a-phase grain size and removal volume, specifically at low current density. This is because the a-phase, which dissolves slowly at low J, (shown previously), is anchored less in the bulk as the grain size is reduced. Thus, the probability of a successful wash-out event is increased as the grain size is reduced. In addition, a finer dispersion of a-phase grains increases the number of discrete potential wash-out events over equivalent surface area. Large $\alpha$-phase grains are retained in the bulk for longer periods, whereby they will eventually be subjected to anodic dissolution, once the field-focussing effects are increased, which occurs as the height differential between the protruding phase and the evolving $\beta$-phase surface increases. This is supported by the optical micrograph of a sectioned pit machined at low current density $\left(20 \mathrm{~A} / \mathrm{cm}^{2}\right)$ into the $650^{\circ} \mathrm{C}$ annealed condition (see Figure 11c), wherein flattened tops of the protruding a-phase grains are visible. This is attributed to field-focussing (described in Figure 8f). In this instance, a-phase removal occurs predominantly through anodic dissolution - not erosion. This is contrasted by the optical micrograph of an equivalent pit in the as-received condition of supply, whereby cratering can be observed and rough topped a-phase grains can be seen (see Figure 
11b). Figure 11a, shows a correlation between removal mass with grain size at low $\left(20 \mathrm{~A} / \mathrm{cm}^{2}\right)$ current density, except for finest-grained sample $\left(800^{\circ} \mathrm{C}-\mathrm{T}\right)$.

For the as-received and $450^{\circ} \mathrm{C}$ annealed conditions, the charge specific removal at low current density is approximately the same as the higher current densities tested, within the range of error. Bhattacharyya (2015), described the expected increase in material removal efficiency with current density in ECM. For $\mathrm{Cu}$ (the main element of this brass), Kinoshita et al. (1970) showed the efficiency to be dependent on the valence state of dissolution. In active modes of dissolution $\left(J \leq 20 \mathrm{~A} / \mathrm{cm}^{2}\right)$, liberated copper species tend towards Cu (II) (cupric). Schneider et al. (2012) demonstrated that increasing the current density into the transpassive regime $\left(J>20 \mathrm{~A} / \mathrm{cm}^{2}\right)$, results in a gradual valence shift towards $\mathrm{Cu}$ (I) (cuprous), requiring a lower charge equivalent for liberation. In light of this expected valence change, it is interesting that the two aforementioned conditions show similar charge-specific removal at all current densities tested. It is possible that this uncharacteristic removal behaviour is a result of erosive removal. As the current density is increased, anodic material removal is also increased, but the contribution from erosion is reduced, reducing expected removal gains. The correlation between grain size and material removal at low current density is broken by the precondition with the smallest measured grain size and basket-weave microstructure $\left(800^{\circ} \mathrm{C}\right.$-tempered). This is thought to be a result of the conjoined nature of the a-phase grains, whereby each grain is anchored into a matrix microstructure, (see Figure 11d). This inhibits discrete wash-out events from occurring during processing, therefore the apparent removal mass is lower. 
For the three largest grained preparations, charge-specific removal in low current density machining is shown to be significantly reduced compared with machining at higher current density (50 and $100 \mathrm{~A} / \mathrm{cm}^{2}$ ), and a more typical relationship between charge-specific removal and current density is observed. This is proposed to be a result of the reduction in the contribution of erosion to the material removal as grain size is reduced, and the phase ratio becomes increasingly skewed. The higher current densities tested show similar values of charge-specific removal generally independent of material precondition, thus the implication is that erosive contributions are either indeterminate or equivalent at these two current density levels, under these testing conditions. Discrete wash-out events are still likely to occur in these processing regimes, however at a lower frequency than at low current density, due to the increase in electropolishing phenomena, which equalise surface asperities, such as protruding a-phase grains.
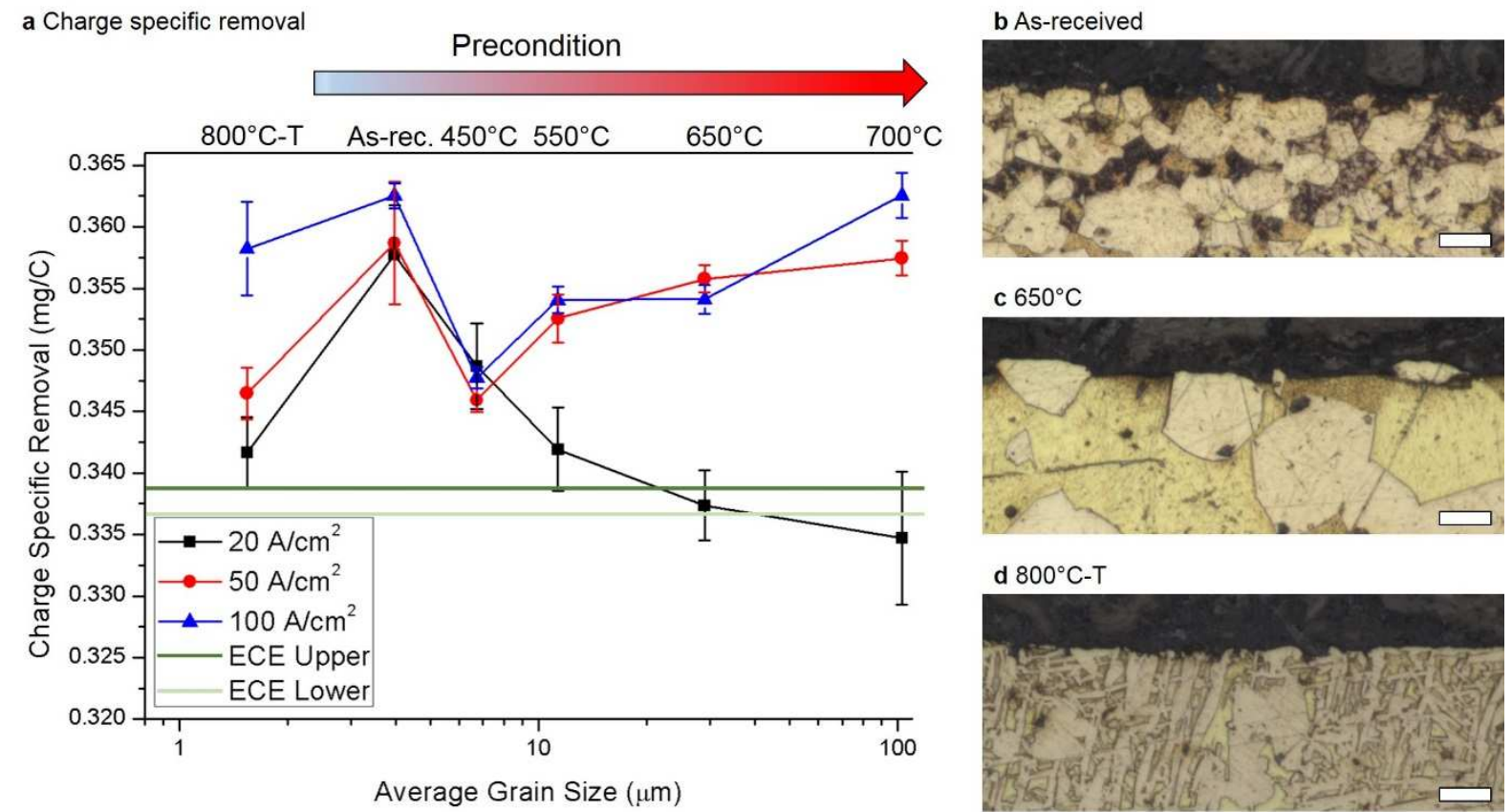

Figure 11: a) Charge specific removal (mg/C), shown for low, medium and high current densities $\left(J_{\text {noz }}=20,100,300 \mathrm{~A} / \mathrm{cm}^{2}\right)$. Sectioned low current density $\left(J_{\text {noz }}=20 \mathrm{~A} / \mathrm{cm}^{2}\right)$ pits machined into b) as-received, c) $650^{\circ} \mathrm{C}$, d) $800^{\circ} \mathrm{C}$ - T conditions of supply. Error bars are the standard deviation from the mean of three discrete pits. Optical scale bars $10 \mu \mathrm{m}$. 


\subsection{Form Observations}

The microstructural contribution to the machined profile in EJP was assessed by appraising charge specific maximum depths for each material precondition, shown in Figure 12a. A positive trend in removal depth can be observed at all tested current densities for the three largest grained preconditions $\left(550,650,700^{\circ} \mathrm{C}\right.$ conditions), where depth increases are shown to be approximately consistent at the current densities tested here $\left(20 \mathrm{~A} / \mathrm{cm}^{2}, 17 \% ; 50 \mathrm{~A} / \mathrm{cm}^{2}, 12 \% ; 100 \mathrm{~A} / \mathrm{cm}^{2}, 13 \%\right)$ across these material conditions. This is exemplified in the cross-sectional pit profiles showing the depth increase across the aforementioned preconditions in Figure $12 \mathrm{~b}$, at $\left(20 \mathrm{~A} / \mathrm{cm}^{2}\right)$. Deeper pits are of particular interest considering the lower removal efficiency of the $700^{\circ} \mathrm{C}$ condition at this current density level, and is suggestive of more precise material removal. Increasing grain size up to $11.3 \mu \mathrm{m}$ (as-received, 450 , and $550^{\circ} \mathrm{C}$ conditions) show no difference in removal depth, within the error range.

The deeper cuts in predominantly single-phase material are proposed to be a result of the ease of machinability of the $\beta$-phase. For such a material, removal occurs unperturbed at the centre of the impingement area, therefore appearing to localise dissolution. Pits therefore become deeper and narrower. A similar phenomenon has been previously reported in the EJP of Ti by Mitchell-Smith and Clare (2016), considering electrochemically inert Ti-containing oxides. For a dual-phase material, the presence of slowly dissolving a-phase partially disrupts removal in the central region, broadening the form for controlled processing parameters, while the depth reduces, despite greater material removal rate for dual-phase materials at low current density. For the finest-grained sample $\left(800^{\circ} \mathrm{C}\right.$-tempered), removal depth is shown to 
increase with current density, a consequence of the large dispersion of a-phase. Removal of this inert and conjoined phase is largely dependent on the relative machinability, $\alpha<\beta$, and thus is closely related to the current density parameter.

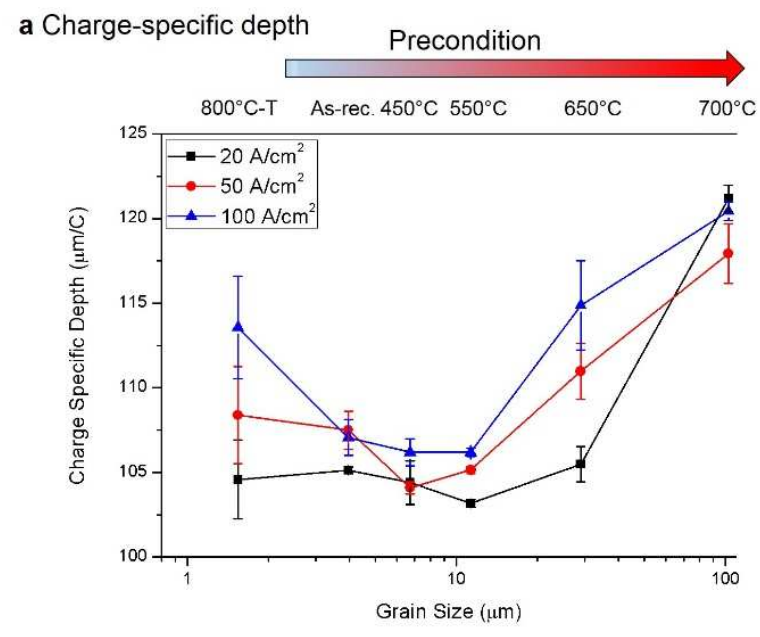

c $450^{\circ} \mathrm{C}, 20 \mathrm{~A} / \mathrm{cm}^{2}$

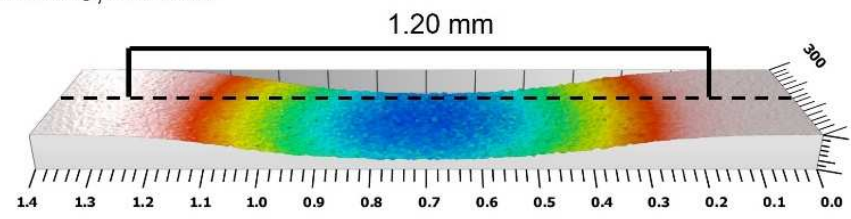

d $700^{\circ} \mathrm{C}, 20 \mathrm{~A} / \mathrm{cm}^{2}$

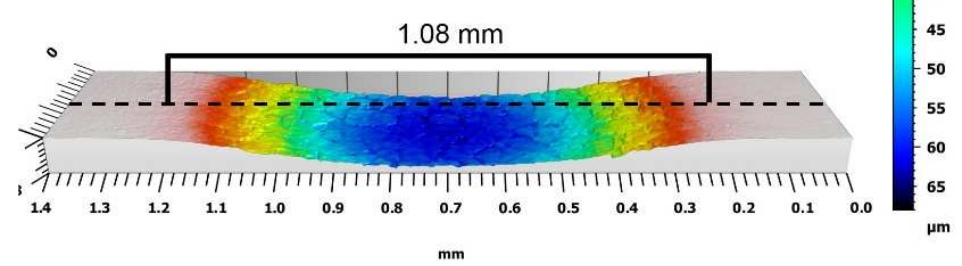

b Profiles, $20 \mathrm{~A} / \mathrm{cm}^{2}$
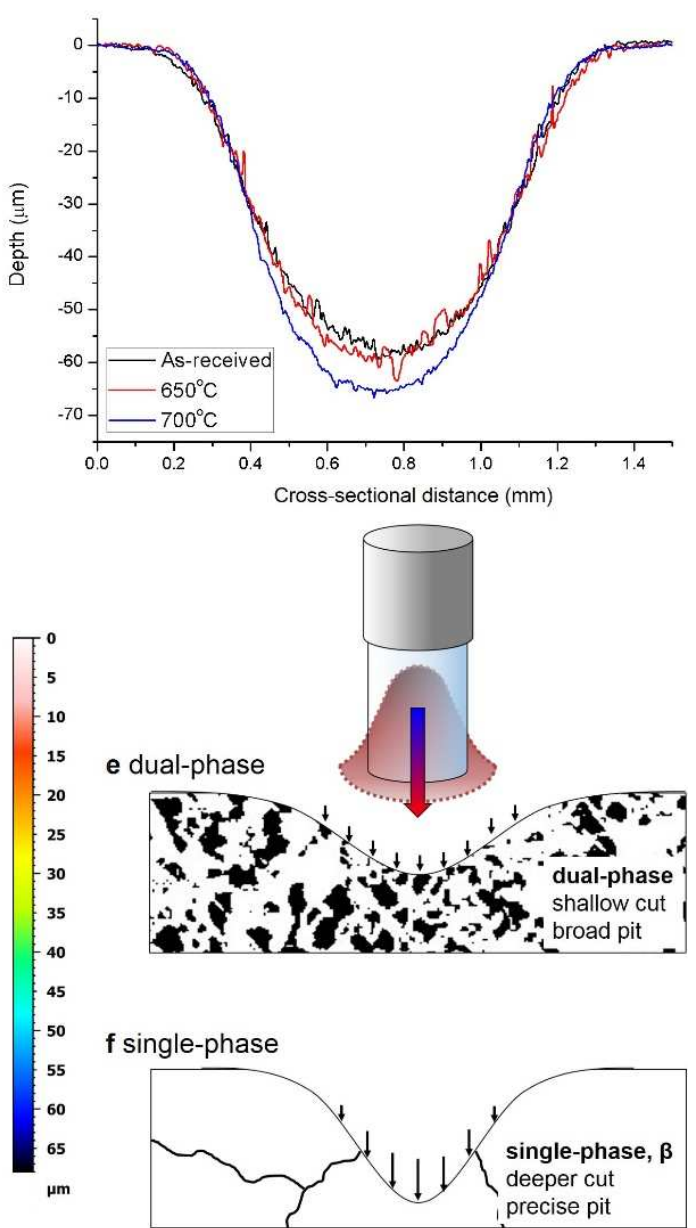

Figure 12: a) Maximum machining depth as a function of material precondition at different current densities $(20,50$ and $100 \mathrm{~A} / \mathrm{cm} 2)$, showing increasing from the $550^{\circ} \mathrm{C}$ to the $700^{\circ} \mathrm{C}$ preconditions. b) Cross-sectional profiles of pits machined at $20 \mathrm{~A} / \mathrm{cm} 2, \mathrm{c}$ ) Reconstruction of a pit machined into the $450^{\circ} \mathrm{C}$ condition, showing a broader, but shallower pit when compared with d), machined in $700^{\circ} \mathrm{C}$ condition. e) Material removal disruption in the central region in a dual-phase material. f) In contrast, unperturbed material removal occurs at the central area exposed to the highest current density, thus broadening is reduced. 


\subsection{Conclusions}

A study considering the surface topography, chemistry, removal mechanisms and resultant form resulting from EJP of a dual-phase brass (Cu39Zn2Pb) has been undertaken. Different material preconditions were appraised in this study, where microstructure significantly influences the aforementioned properties and must be considered to facilitate achievement of design intent.

Predominantly single-phase materials result in smoother surfaces. Increasing a-phase grain size increases surface height at low current density in point machining and at all current densities in striation machining, resulting from anchoring of larger grains. In the latter case, two relationships are observed as grain size is increased: i) increasing step height increases roughness when the phase ratio is unpolarised. ii) Conversely, as the phase ratio polarises and the number of discrete steps decreases, the surface amplitude decreases, despite increasing step height.

Differential machinability between phases reduces upon increasing current density. In addition, the chemistry of the near surface is significantly altered as $\mathrm{Zn}$ is leeched preferentially over $\mathrm{Cu}$, occurring more prolifically at low current density. Dezincification results in a Cu-rich boundary layer, of increasing resistance to anodic dissolution. Surface metallurgy can however be ensured through increasing current density, such that the resulting surface composition is consistent with the bulk material. 
Erosive removal has been demonstrated in EJP for non-ceramic $\alpha$-phase crystallites, resulting from intergranular attack, which occurs through undermining of the a-phase grains. These are washed away in the impinging electrolyte jet, leaving recesses consistent with a-phase grain size. This occurs mainly at low current densities, resulting in a decrease in removal rate with increasing grain size $(6 \%$ decrease in single-phase compared with as-received condition). Larger grain sizes are more resistant to wash-out and are retained at the surface, where they are attacked electrochemically as a result of field-focussing effects.

Machined feature form has been shown to depend on phase ratio. For predominantly single-phase materials, machining becomes more precise and cuts are deeper than for dual-phase preconditions. This is understood to be a result of removal occurring in an unperturbed manner at jet core, whereas for a dual-phase system, inert grains disrupt central removal, broadening the feature and decreasing the depth.

Therefore, appropriate EJP parameters depend on the intent and the microstructure. For smooth topographies and surface metallurgy representative of the bulk material, higher current densities are required. EJP of single-phase alloys benefits from enhanced dimensional accuracy, smoother topography and greater depth of cut. Where component microstructures can be designed prior to EJP, for example additively manufactured parts, single-phase materials are desirable for decreasing final roughness in EJP, whereas material removal at low current density is enhanced for small-grained dual-phase materials. 


\section{Acknowledgements}

This work was supported by the Engineering and Physical Sciences Research Council [grant numbers EP/M02072X/1, EP/L016206/1] through the "In-Jet Interferometry for Ultra Precise Electrolyte Jet Machining" project, and the EPSRC Centre for Doctoral Training in Innovative Metal Processing. The authors would like to thank the Manufacturing Metrology Team and the Nanoscale and Microscale Research Centre at the University of Nottingham for the use of equipment, and Alexander Jackson-Crisp (ACEL) for help with sample preparation.

\section{Author Contributions}

A.S. conceived the study. A.S., J.M.S. and I.B. performed experimentation. A.T.C. guided the research. A.S. analysed the data. A.S. wrote the manuscript with help from all authors. All authors approved the final manuscript. 


\section{References}

Bhattacharyya, B., 2015. Electrochemical Micromachining for Nanofabrication, MEMS and Nanotechnology. William Andrew Publishing, New York, pp. 53-67.

Burger, M., Koll, L., Werner, E.A., Platz, A., 2012, Electrochemical machining characteristics and resulting surface quality of the nickel-base single-crystalline material LEK94. Journal of Manufacturing Processes 14, 62-70.

García, P., Rivera, S., Palacios, M., Belzunce, J., 2010. Comparative study of the parameters influencing the machinability of leaded brasses. Engineering Failure Analysis 17, 771-776.

Hackert-Oschätzchen, M., Lehnert, N., Kowalick, M., Scherf, C., Martin, A., Schubert, A., 2016. Analysis of an Electrochemical Machining Process for Particle Reinforced Aluminium Matrix Composites. 2016 COMSOL Conference, Munich.

Hackert-Oschätzchen, M., Martin, A., Meichsner, G., Zinecker, M., Schubert, A., 2013. Microstructuring of carbide metals applying Jet Electrochemical Machining. Precision Engineering 37, 621-634.

Hackert-Oschätzchen, M., Meichsner, G., Zinecker, M., Martin, A., Schubert, A., 2012. Micro machining with continuous electrolytic free jet. Precision Engineering 36, 612-619.

Haisch, T., Mittemeijer, E.J., Schultze, J.W., 2004. High rate anodic dissolution of $100 \mathrm{Cr} 6$ steel in aqueous $\mathrm{NaNO}_{3}$ solution. Journal of Applied Electrochemistry 34, 997-1005.

Humphreys, F.J., Hatherly, M., 2004. Recrystallization and Related Annealing Phenomena, 2nd ed. Elsevier Ltd, Oxford.

Ippolito, R., Tornincasa, S., Capello, G., Micheletti, G.F., 1981. Electron-Jet Drilling - Basic Involved Phenomena. CIRP Annals - Manufacturing Technology 30, 87-90.

Kawanaka, T., Kunieda, M., 2015. Mirror-like finishing by electrolyte jet machining. CIRP Annals - Manufacturing Technology 64, 237-240.

Kinoshita, K., Landolt, D., Muller, R.H., Tobias, C.W., 1970. Stoichiometry of Anodic Copper Dissolution at High Current Densities. Journal of The Electrochemical Society 117, 1246-1251.

Klocke, F., Harst, S., Ehle, L., Zeis, M., Klink, A., 2018. Surface integrity in electrochemical machining processes: An analysis on material modifications occuring during electrochemical machining. Proceedings of the Institution of Mechanical Engineers Part B 232 (4), 578-585.

Kozak, J., Rajurkar, K.P., Balkrishna, R., 1996. Study of Electrochemical Jet Machining Process. Journal of Manufacturing Science and Engineering 118, 490-498.

Kozak, J., Zybura-Skrabalak, M., 2016. Some Problems of Surface Roughness in Electrochemical Machining (ECM). Procedia CIRP 42, 101-106.

Krishnaiah Chetty, O.V., Radhakrishnan, V., 1981. A study on the influence of grain size in electrochemical machining. International Journal of Machine Tool Design and Research 21, 57-69.

Kunieda, M., Okada, A., Furutani, K., Miyoshi, K., Kunieda, M., 2016. Fabrication of Micro Rods of Cemented Carbide by Electrolyte Jet Turning. Procedia CIRP 42, 373-378.

Lange, N.A., 1999. Lange's Handbook of Chemistry, 15th ed. McGraw-Hill, New York.

Leach, R., 2011. Optical Measurement of Surface Topography. Springer-Verlag, Berlin.

Mitchell-Smith, J., Clare, A.T., 2016. ElectroChemical Jet Machining of Titanium: Overcoming Passivation Layers with Ultrasonic Assistance. Procedia CIRP 42, 379-383.

Mitchell-Smith, J., Speidel, A., Clare, A.T., 2018a. Advancing electrochemical jet methods through manipulation of the angle of address. Journal of Materials Processing Technology 255, 364-372.

Mitchell-Smith, J., Speidel, A., Clare, A.T., 2018b. Transitory electrochemical masking for precision jet processing techniques. Journal of Manufacturing Processes 31, 273-285.

Natsu, W., Ikeda, T., Kunieda, M., 2007. Generating complicated surface with electrolyte jet machining. Precision Engineering 31, 33-39.

Nunes, R., Adams, J.H., Ammons, M., Avery, H.S., Barnhurst, R.J., Bean, J.C., Beaudry, B.J., 1979. Properties and Selection: Nonferrous Alloys and Pure Metals. ASM International.

Pickering, H.W., Byrne, P.J., 1969. Partial Currents During Anodic Dissolution of Cu-Zn Alloys at Constant Potential. Journal of The Electrochemical Society 116, 1492-1496.

Pickering, H.W., Wagner, C., 1967. Electrolytic Dissolution of Binary Alloys Containing a Noble Metal. Journal of The Electrochemical Society 114, 698-706.

Schneider, M., Schroth, S., Richter, S., Höhn, S., Schubert, N., Michaelis, A., 2012. In-situ investigation of the interplay between microstructure and anodic copper dissolution under near-ECM conditions-Part 2: The transpassive state. Electrochimica Acta 70, 76-83.

Speidel, A., Lutey, A.H.A., Mitchell-Smith, J., Rance, G.A., Liverani, E., Ascari, A., Fortunato, A., Clare, A., 2016. Surface modification of mild steel using a combination of laser and electrochemical processes. Surface and Coatings Technology.

Walker, P., Tarn, W., 1991. CRC Handbook of Metal Etchants. CRC Press, USA

Wang, X., Qu, N., Guo, P., Fang, X., Lin, X., 2017. Electrochemical Machining Properties of the Laser Rapid Formed Inconel 718 Alloy in $\mathrm{NaNO}_{3}$ Solution. Journal of the Electrochemical Society 164 (14), E548-E559.

White, F.M., 2010. Fluid Mechanics, 7th ed. The McGraw-Hill Companies, Inc., New York.

Yoneda, K., Kunieda, M., 1995. Numerical Analysis of Cross Sectional Shape of Micro-Indents Formed by the Electrochemical Jet Machining (ECJM). Journal of The Japan Society of Electrical Machining Engineers 29, 1-8. 
